\title{
Sparse Sums of Positive Semidefinite Matrices
}

\author{
Marcel K. de Carli Silva* $\quad$ Nicholas J. A. Harvey ${ }^{\dagger} \quad$ Cristiane M. Sato $^{\ddagger}$
}

\begin{abstract}
Recently there has been much interest in "sparsifying" sums of rank one matrices: modifying the coefficients such that only a few are nonzero, while approximately preserving the matrix that results from the sum. Results of this sort have found applications in many different areas, including sparsifying graphs. In this paper we consider the more general problem of sparsifying sums of positive semidefinite matrices that have arbitrary rank.

We give several algorithms for solving this problem. The first algorithm is based on the method of Batson, Spielman and Srivastava (2009). The second algorithm is based on the matrix multiplicative weights update method of Arora and Kale (2007). We also highlight an interesting connection between these two algorithms.

Our algorithms have numerous applications. We show how they can be used to construct graph sparsifiers with auxiliary constraints, sparsifiers of hypergraphs, and sparse solutions to semidefinite programs.
\end{abstract}

\footnotetext{
${ }^{*}$ Department of Combinatorics and Optimization, University of Waterloo. mksilva@uwaterloo.ca. Partially supported by an NSERC Discovery Grant of L. Tunçel.

${ }^{\dagger}$ Department of Computer Science, University of British Columbia. nickhar@cs.ubc.ca. Supported by an NSERC Discovery Grant.

${ }^{\ddagger}$ Department of Combinatorics and Optimization, University of Waterloo. cmsato@uwaterloo.ca. Partially supported by an NSERC Discovery Grant of N. Wormald.
} 


\section{Introduction}

A sparsifier of a graph is a subgraph that approximately preserves some structural properties of the graph. The original work in this area studied cut sparsifiers, which are weighted subgraphs that approximate every cut arbitrarily well. The celebrated work of Benczúr and Karger [5, 6] proved that every undirected graph with $n$ vertices and $m$ edges (and potentially non-negative weights on its edges) has a subgraph with only $O\left(n \log n / \varepsilon^{2}\right)$ edges (and new weights on those edges) such that, for every cut, the weight of the cut in the original graph and its subgraph agree up to a multiplicative factor of $(1 \pm \varepsilon)$. Benczúr and Karger also gave a randomized algorithm to construct a cut sparsifier in $\tilde{O}\left(m / \varepsilon^{2}\right)$ time. Recent work has extended and improved their algorithm in various ways [10, 11, 12, 14, 15].

Spielman and Teng [39] introduced spectral sparsifiers, which are weighted subgraphs such that the quadratic forms defined by the Laplacians of the graph and the sparsifier agree up to a multiplicative factor of $(1 \pm \varepsilon)$. Spectral sparsifiers are also cut sparsifiers, as can be seen by evaluating these quadratic forms at $\{0,1\}$-vectors. They proved that every undirected graph with $n$ vertices and $m$ edges (and potentially nonnegative weights on its edges) has a spectral sparsifier with only $n$ polylog $(n) / \varepsilon^{2}$ edges (and new weights on those edges). Spielman and Srivastava [38] reduce the graph sparsification problem to the following abstract problem in matrix theory.

Problem 1. Let $v_{1}, \ldots, v_{m} \in \mathbb{R}^{n}$ be vectors and let $B=\sum_{i} v_{i} v_{i}^{T}$. Given $\varepsilon \in(0,1)$, find a vector $y \in \mathbb{R}^{m}$ with small support such that $y \geq 0$ and

$$
B \preceq \sum_{i} y_{i} v_{i} v_{i}^{T} \preceq(1+\varepsilon) B .
$$

(Here the notation $X \preceq Y$ means that the matrix $Y-X$ is positive semidefinite.)

Spielman and Srivastava [38] observe that Problem 1 can be solved using known concentration bounds on operator-valued random variables, specifically Rudelson's sampling lemma [32, 33]. This approach yields a vector $y$ with support size $O\left(n \log n / \varepsilon^{2}\right)$, and therefore yields a construction of spectral sparsifiers with $O\left(n \log n / \varepsilon^{2}\right)$ edges. Their algorithm relies on the linear system solver of Spielman and Teng [39], which was significantly simplified by Koutis, Miller and Peng [24]. Recent work [23] has improved the space usage of Spielman and Srivastava's algorithm.

In subsequent work, Batson, Spielman and Srivastava [4] give a deterministic algorithm that solves Problem 1 and produces a vector $y$ with support size $O\left(n / \varepsilon^{2}\right)$. Consequently they obtain improved spectral sparsifiers with $O\left(n / \varepsilon^{2}\right)$ edges. This work led to important progress in metric embeddings [29, 34], convex geometry [40] and Banach space theory [37].

In this paper, we focus on a more general problem.

Problem 2. Let $B_{1}, \ldots, B_{m}$ be symmetric, positive semidefinite matrices of size $n \times n$ and let $B=\sum_{i} B_{i}$. Given $\varepsilon \in(0,1)$, find a vector $y \in \mathbb{R}^{m}$ with small support such that $y \geq 0$ and

$$
B \preceq \sum_{i} y_{i} B_{i} \preceq(1+\varepsilon) B .
$$

This problem can also be solved by known concentration bounds: Ahlswede and Winter [1] give a method for generalizing Chernoff-like bounds to operator-valued random variables, and one of their theorems [1, Theorem 19] directly yields a solution to Problem 2] (Other expositions of these results also exist [41, 16].) This approach yields a vector $y$ with support size $O\left(n \log n / \varepsilon^{2}\right)$. See Section 3 for more details.

This paper gives two improved solutions to Problem 2, Our interest in this topic is motivated by several applications, such as constructing sparsifiers with certain auxiliary properties and sparsifiers for hypergraphs. We discuss these applications in Section 1.2. 


\subsection{Our Results}

We give several efficient algorithms for solving Problem 2 , Our strongest solution is:

Theorem 3. Let $B_{1}, \ldots, B_{m}$ be symmetric, positive semidefinite matrices of size $n \times n$ and arbitrary rank. Set $B:=\sum_{i} B_{i}$. For any $\varepsilon \in(0,1)$, there is a deterministic algorithm to construct a vector $y \in \mathbb{R}^{m}$ with $O\left(n / \varepsilon^{2}\right)$ nonzero entries such that $y \geq 0$ and

$$
B \preceq \sum_{i} y_{i} B_{i} \preceq(1+\varepsilon) B .
$$

The algorithm runs in $O\left(m n^{3} / \varepsilon^{2}\right)$ time. Moreover, the result continues to hold if the input matrices $B_{1}, \ldots, B_{m}$ are Hermitian and positive semidefinite.

Our proof of Theorem 3 is quite simple and builds on results of Batson, Spielman and Srivastava [4]. We remark that the assumption that the $B_{i}$ 's are positive semidefinite cannot be removed; see Appendix D]

We also give a second solution to Problem 2 which is quantitatively weaker, although it is based on very general machinery which might prove useful in further applications or generalizations of Problem 2 This second solution is based on the matrix multiplicative weights update method (MMWUM) of Arora and Kale [3, 22]. By a black-box application of their theorems we obtain a deterministic algorithm to construct a vector $y$ with $O\left(n \log n / \varepsilon^{3}\right)$ nonzero entries. By slightly refining their analysis we can improve the number of nonzero entries to $O\left(n \log n / \varepsilon^{2}\right)$. We remark that Orecchia and Vishnoi [30] have used MMWUM for solving the balanced separator problem; this can be used as a subroutine in Spielman and Teng's algorithm for constructing spectral sparsifiers.

Another virtue of our second solution is that it illustrates that the surprising Batson-Spielman-Srivastava (BSS) algorithm is actually closely related to MMWUM. In particular, the algorithms underlying our two solutions are identical, except for the use of slightly different potential functions. We explain this connection in Section 8

\subsection{Applications}

In this section, we present several applications of Problem 2 Proofs are given in Appendix A

\section{Sparsifiers with costs.}

Corollary 4. Let $G=(V, E)$ be a graph, let $w: E \rightarrow \mathbb{R}_{+}$be a weight function, and let $c_{1}, \ldots, c_{k}: E \rightarrow \mathbb{R}_{+}$ be cost functions, with $k=O(n)$. Let $\mathcal{L}_{G}(w)$ denote the Laplacian matrix for graph $G$ with weight function $w$. For any real $\varepsilon \in(0,1)$, there is a deterministic polynomial-time algorithm to find a subgraph $H$ of $G$ and a weight function $w_{H}: E(H) \rightarrow \mathbb{R}_{+}$such that

$$
\begin{gathered}
\mathcal{L}_{G}(w) \preceq \mathcal{L}_{H}\left(w_{H}\right) \preceq(1+\varepsilon) \mathcal{L}_{G}(w), \\
\sum_{e \in E} w_{e} c_{i, e} \leq \sum_{e \in E(H)} w_{H, e} c_{i, e} \leq(1+\varepsilon) \sum_{e \in E} w_{e} c_{i, e} \quad \text { for all } i
\end{gathered}
$$

and $|E(H)|=O\left(n / \varepsilon^{2}\right)$.

The inequalities $\mathcal{L}_{G}(w) \preceq \mathcal{L}_{H}\left(w_{H}\right) \preceq(1+\varepsilon) \mathcal{L}_{G}(w)$ are equivalent to the condition that the subgraph $H$ (with weights $w_{H}$ ) is a spectral sparsifier of $G$ (with weights $w$ ). We remark that existing methods for producing sparsifiers have low probability of approximately satisfying even a single cost function (i.e., the case $k=1$ ).

One potentially interesting application of sparsifiers with costs is as follows. 
Corollary 5 (Rainbow Sparsifiers). Let $G=(V, E)$ be a graph and let $w: E \rightarrow \mathbb{R}_{+}$be a weight function. Let $E_{1}, \ldots, E_{k}$ be a partition of the edges, i.e., each edge is colored with one of $k$ colors. For any real $\varepsilon \in(0,1)$, there is a deterministic polynomial-time algorithm to find a subgraph $H$ of $G$ and a weight function $w_{H}: E(H) \rightarrow \mathbb{R}_{+}$such that

$$
\begin{aligned}
\mathcal{L}_{G}(w) \preceq \mathcal{L}_{H}\left(w_{H}\right) & \preceq(1+\varepsilon) \mathcal{L}_{G}(w), \\
(1-\varepsilon) \sum_{e \in E_{i}} w_{e} \leq \sum_{e \in E(H) \cap E_{i}} w_{H, e} & \leq(1+\varepsilon) \sum_{e \in E_{i}} w_{e} \quad \text { for all } i,
\end{aligned}
$$

and $|E(H)|=O\left((n+k) / \varepsilon^{2}\right)$.

Hypergraph sparsifiers. Let $\mathcal{H}=(V, \mathcal{E})$ be a hypergraph, and let $w: \mathcal{E} \rightarrow \mathbb{R}_{+}$. We follow the definition of Laplacian for hypergraphs as in [31]. For each hyperedge $E \in \mathcal{E}$, define its Laplacian $\mathcal{L}_{E}$ as the graph Laplacian of a graph on $V$ whose edge set forms a clique on $E$. Define the Laplacian for the hypergraph $\mathcal{H}$ with weight function $w$ as the matrix $\mathcal{L}_{\mathcal{H}}(w):=\sum_{E \in \mathcal{E}} w_{E} \mathcal{L}_{E}$.

Corollary 6 (Spectral sparsifiers for hypergraphs). For any real $\varepsilon \in(0,1)$, there is a deterministic polynomialtime algorithm to find a sub-hypergraph $\mathcal{G}$ of $\mathcal{H}$ and a weight function $w_{\mathcal{G}}: \mathcal{E}(\mathcal{G}) \rightarrow \mathbb{R}_{+}$such that

$$
\mathcal{L}_{\mathcal{H}}(w) \preceq \mathcal{L}_{\mathcal{G}}\left(w_{\mathcal{G}}\right) \preceq(1+\varepsilon) \mathcal{L}_{\mathcal{H}}(w),
$$

and $|\mathcal{E}(\mathcal{G})|=O\left(n / \varepsilon^{2}\right)$.

This corollary concerns spectral sparsifiers. It is also interesting to study sparsifiers that approximately preserve all cuts. There are several ways to extend the definition of "the weight of a cut" from ordinary graphs to hypergraphs. We consider the following two definitions, where $S$ is any set of vertices in a hypergraph $\mathcal{H}$ with edge weights $w$.

- $w\left(\delta_{\mathcal{H}}(S)\right)$ : This is the sum of the weights of all hyperedges that contain at least one vertex in $S$ and at least one vertex in $\bar{S}:=V \backslash S$.

- $w^{*}\left(\delta_{\mathcal{H}}(S)\right)$ : This is defined to be $\sum_{E \in \mathcal{E}} w_{E} \cdot|S \cap E| \cdot|\bar{S} \cap E|$.

Obviously these definitions agree in ordinary graphs.

Corollary 7 (Cut sparsifiers for hypergraphs, second definition). For any real $\varepsilon \in(0,1)$, there is a deterministic polynomial-time algorithm to find a sub-hypergraph $\mathcal{G}$ of $\mathcal{H}$ and a weight function $w_{\mathcal{G}}: \mathcal{E}(\mathcal{G}) \rightarrow \mathbb{R}_{+}$ such that

$$
w^{*}\left(\delta_{\mathcal{H}}(S)\right) \leq w_{\mathcal{G}}^{*}\left(\delta_{\mathcal{G}}(S)\right) \leq(1+\varepsilon) w^{*}\left(\delta_{\mathcal{H}}(S)\right) \quad \text { for every } S \subseteq V
$$

and $|\mathcal{E}(\mathcal{G})|=O\left(n / \varepsilon^{2}\right)$.

Corollary 8 (Cut sparsifiers for hypergraphs, first definition). Assume that $\mathcal{H}$ is an r-uniform hypergraph. For any real $\varepsilon \in(0,1)$, there is a deterministic polynomial-time algorithm to find a sub-hypergraph $\mathcal{G}$ of $\mathcal{H}$ and a weight function $w_{\mathcal{G}}: \mathcal{E}(\mathcal{G}) \rightarrow \mathbb{R}_{+}$such that

$$
\frac{(r-1)}{r^{2} / 4} w\left(\delta_{\mathcal{H}}(S)\right) \leq w_{\mathcal{G}}\left(\delta_{\mathcal{G}}(S)\right) \leq \frac{(1+\varepsilon) r^{2}}{4(r-1)} w\left(\delta_{\mathcal{H}}(S)\right) \quad \forall S \subseteq V
$$

and $|\mathcal{E}(\mathcal{G})|=O\left(n / \varepsilon^{2}\right)$. In other words, the sparsified hypergraph $\mathcal{G}$ approximates the weight of the cuts in the hypergraph $\mathcal{H}$ to within a factor $\Theta\left(r^{2}\right)$. 
For the special case $r=3$, we can achieve $(1+\varepsilon)$-approximate sparsification for all cuts, even under the first definition.

Corollary 9 (Cut sparsifiers for 3-uniform hypergraphs). Assume that $\mathcal{H}$ is a 3-uniform hypergraph. For any $\varepsilon \in(0,1)$, there is a deterministic polynomial-time algorithm to find a sub-hypergraph $\mathcal{G}$ of $\mathcal{H}$ and $a$ weight function $w_{\mathcal{G}}: \mathcal{E}(\mathcal{G}) \rightarrow \mathbb{R}_{+}$such that

$$
w\left(\delta_{\mathcal{H}}(S)\right) \leq w_{\mathcal{G}}\left(\delta_{\mathcal{G}}(S)\right) \leq(1+\varepsilon) w\left(\delta_{\mathcal{H}}(S)\right) \quad \forall S \subseteq V
$$

and $|\mathcal{E}(\mathcal{G})|=O\left(n / \varepsilon^{2}\right)$.

\section{Sparse solutions to semidefinite programs.}

Corollary 10. Let $A_{1}, \ldots, A_{m}$ be symmetric, positive semidefinite matrices of size $n \times n$, and let $B$ be a symmetric matrix of size $n \times n$. Let $c \in \mathbb{R}^{m}$ with $c \geq 0$. Suppose that the semidefinite program (SDP)

$$
\min \left\{c^{T} z: \sum_{i} z_{i} A_{i} \succeq B, z \in \mathbb{R}^{m}, z \geq 0\right\}
$$

has a feasible solution $z^{*}$. Then, for any real $\varepsilon \in(0,1)$, it has a feasible solution $\bar{z}$ with at most $O\left(n / \varepsilon^{2}\right)$ nonzero entries and $c^{T} \bar{z} \leq(1+\varepsilon) c^{T} z^{*}$.

Several important SDPs can be cast as in Corollary 10, see, e.g., [19, 20]. Recently, Jain and Yao [21] gave a parallel approximation algorithm for SDPs in this form with $B$ positive semidefinite.

Lovász theta number. For a graph $G=(V, E)$ on $n$ nodes, let $t^{\prime}(G)$ denote the square of the minimum radius of an Euclidean ball in $\mathbb{R}^{n}$ such that there is a map from $V$ to points in the ball such that adjacent vertices are mapped to points at distance at least 1 . Also, let $\vartheta^{\prime}(G)$ denote the variant of the Lovász theta number introduced in [27] and [35].

Corollary 11. Let $G=(V, E)$ be a graph. For any real $\varepsilon \in(0,1)$, there is a deterministic polynomial-time algorithm to find a subgraph $H$ of $G$ such that

$$
(1-\varepsilon) t^{\prime}(G) \leq t^{\prime}(H) \leq t^{\prime}(G)
$$

and $|E(H)|=O\left(n / \varepsilon^{2}\right)$.

Corollary 12. Let $G=(V, E)$ be a graph. For any real $\varepsilon \in(0,1)$, there is a deterministic polynomial-time algorithm to find a supergraph $H$ of $G$ such that

$$
\frac{\vartheta^{\prime}(G)}{1-\varepsilon+\varepsilon \vartheta^{\prime}(G)} \leq \vartheta^{\prime}(H) \leq \vartheta^{\prime}(G)
$$

and $|E(H)|=\left(\begin{array}{l}n \\ 2\end{array}\right)-O\left(n / \varepsilon^{2}\right)$.

Corollary 13. Let $G$ be a graph such that $\vartheta^{\prime}(G)=o(\sqrt{n})$. For any real $\gamma>0$, there is a supergraph $H$ of G such that

$$
\frac{\vartheta^{\prime}(G)}{1+\gamma} \leq \vartheta^{\prime}(H) \leq \vartheta^{\prime}(G)
$$

and $|E(H)|=\left(\begin{array}{l}n \\ 2\end{array}\right)-O\left(n \vartheta(G)^{2} / \gamma^{2}\right)$.

Corollary 14. Let $G$ be a graph such that $\vartheta^{\prime}(G)=\Omega(\sqrt{n})$. For any real $\gamma \geq 1$, there is a supergraph $H$ of G such that

$$
\vartheta^{\prime}(H)=\Omega(\sqrt{n} / \gamma)
$$

and $|E(H)|=\left(\begin{array}{l}n \\ 2\end{array}\right)-O\left(n^{2} / \gamma^{2}\right)$. 
Approximate Carathéodory theorems. One immediate application for Theorem 3 is an approximate Carathéodory-type theorem. A classic result of this sort is:

Theorem 15 (Althöfer [2], Lipton-Young [25]). Let $v_{1}, \ldots, v_{m} \in[0,1]^{n}$ and let $\lambda \in \mathbb{R}^{m}$ satisfy $\lambda \geq 0$ and $\sum_{i} \lambda_{i}=1$. Then there exists $\mu \in \mathbb{R}^{m}$ with $\mu \geq 0, \sum_{i} \mu_{i}=1$ and only $O\left(\log n / \varepsilon^{2}\right)$ nonzero entries such that $\left\|\sum_{i} \lambda_{i} v_{i}-\sum_{i} \mu_{i} v_{i}\right\|_{\infty} \leq \varepsilon$.

This theorem follows from simple random sampling arguments, but it has several interesting consequences, including the existence of sparse, low-regret solutions to zero-sum games. The following corollary of Theorem 3 can be viewed as a matrix generalization of Theorem 15 .

Corollary 16. Let $B_{1}, \ldots, B_{m}$ be symmetric, positive semidefinite matrices of size $n \times n$ and let $\lambda \in \mathbb{R}^{m}$ satisfy $\lambda \geq 0$ and $\sum_{i} \lambda_{i}=1$. Let $B=\sum_{i} \lambda_{i} B_{i}$. For any $\varepsilon \in(0,1)$, there exists $\mu \geq 0$ with $\sum_{i} \mu_{i}=1$ such that $\mu$ has $O\left(n / \varepsilon^{2}\right)$ nonzero entries and

$$
(1-\varepsilon) B \preceq \sum_{i} \mu_{i} B_{i} \preceq(1+\varepsilon) B .
$$

Although the support size in Theorem 15 is much smaller than in Corollary 16, the latter provides a multiplicative error bound whereas the former only provides an additive error bound. Theorem 15 can be modified to give multiplicative error bounds if we allow $\mu$ to have $O\left(n \log n / \varepsilon^{2}\right)$ non-zero entries. However such a result is not interesting as Carathéodory's theorem provides a $\mu$ with only $n+1$ non-zero entries and no error (i.e., $\epsilon=0$ ). In contrast, Carathéodory's theorem is very weak in the scenario of Corollary 16 as it only provides a $\mu$ with $n(n+1) / 2+1$ nonzero entries.

\section{Sparsifiers on subgraphs.}

Corollary 17. Let $G=(V, E)$ be a graph, let $w: E \rightarrow \mathbb{R}_{+}$be a weight function, and let $\mathcal{F}$ be a collection of subgraphs of $G$ such that $\sum_{F \in \mathcal{F}}|V(F)|=O(n)$. For any real $\varepsilon \in(0,1)$, there is a deterministic polynomial-time algorithm to find a subgraph $H$ of $G$ and a weight function $w_{H}: E(H) \rightarrow \mathbb{R}_{+}$such that $|E(H)|=O\left(n / \varepsilon^{2}\right)$ and

$$
\begin{gathered}
\mathcal{L}_{G}(w) \preceq \mathcal{L}_{H}\left(w_{H}\right) \preceq(1+\varepsilon) \mathcal{L}_{G}(w), \\
\mathcal{L}_{F}\left(w_{F}\right) \preceq \mathcal{L}_{H \cap F}\left(w_{H} \uparrow_{E(H \cap F)} \preceq(1+\varepsilon) \mathcal{L}_{F}\left(w_{F}\right) \quad \text { for all } F \in \mathcal{F},\right.
\end{gathered}
$$

where $w_{F}:=w \uparrow_{E(F)}$ is the restriction of $w$ to the coordinates $E(F)$ and $H \cap F=(V(F), E(F) \cap E(H))$.

\section{Preliminaries}

For a non-negative integer $n$, we denote $[n]:=\{1, \ldots, n\}$. The non-negative reals are denoted by $\mathbb{R}_{+}$. The set of $n \times n$ symmetric matrices is denoted by $\mathbb{S}^{n}$. The set of symmetric, $n \times n$ positive semidefinite (resp., positive definite) matrices is denoted by $\mathbb{S}_{+}^{n}$ (resp., $\mathbb{S}_{++}^{n}$ ). Recall that $X \in \mathbb{S}^{n}$ is positive semidefinite if $v^{T} X v \geq 0$ for all $v \in \mathbb{R}^{n}$, and $X$ is positive definite if $X$ is positive semidefinite and $v^{T} X v=0$ implies $v=0$. Sometimes we denote $X \in \mathbb{S}_{+}^{n}$ by $X \succeq 0$ and the notation $X \succeq Y$ means that $X-Y \succeq 0$. For $X \in \mathbb{S}^{n}$ and $a, b \in \mathbb{R}$, the notation $X \in[a, b]$ means that $a I \preceq X \preceq b I$, where $I$ is the identity matrix.

For $X \in \mathbb{S}^{n}$, its trace is $\operatorname{Tr} X:=\sum_{i=1}^{n} X_{i i}$, its largest (resp., smallest) eigenvalue is denoted by $\lambda_{\max }(X)$ (resp., $\lambda_{\min }(X)$ ). The vector space $\mathbb{S}^{n}$ can be endowed with the trace inner product $\langle\cdot, \cdot\rangle$ defined by $\langle X, Y\rangle:=\operatorname{Tr}(X Y)=\sum_{i, j} X_{i j} Y_{i j}$ for every $X, Y \in \mathbb{S}^{n}$. We shall repeatedly use that $\operatorname{Tr}(X Y)=\operatorname{Tr}(Y X)$ for any matrices $X, Y$ for which the products $X Y$ and $Y X$ make sense. 
Let $G=(V, E)$ be a graph. The canonical basis vectors of $\mathbb{R}^{V}$ are $\left\{e_{i}: i \in V\right\}$, and the canonical basis vectors of $\mathbb{R}^{E}$ are $\left\{e_{\{i, j\}}:\{i, j\} \in E\right\}$. The Laplacian of $G$ is the linear transformation $\mathcal{L}_{G}(\cdot): \mathbb{R}^{E} \rightarrow \mathbb{S}^{V}$ defined by $\mathcal{L}_{G}(w)=\sum_{\{i, j\} \in E} w_{\{i, j\}}\left(e_{i}-e_{j}\right)\left(e_{i}-e_{j}\right)^{T}$.

When dealing with Problem 2, we may assume that $B=I$. See [4, Proof of Theorem 1.1] for the details of the reduction.

\section{Solving Problem 2 by Ahlswede-Winter}

As mentioned earlier, Spielman and Srivastava [38] explain how Problem 1 can be solved by Rudelson's sampling lemma. This lemma can be easily generalized to handle matrices of arbitrary rank using the Ahlswede-Winter inequality, yielding a solution to Problem 2

Let $X$ be a random matrix such that $X=B_{i} / \operatorname{Tr} B_{i}$ with probability $p_{i}:=\operatorname{Tr} B_{i} / \operatorname{Tr} I$. Since $B_{i} \succeq 0$ and $\sum_{i} B_{i}=I$, the $p_{i}$ 's define a probability distribution.

Theorem 18 ([1, Theorem 19]). Let $X, X_{1}, \ldots, X_{T}$ be i.i.d. random variables with values in $\mathbb{S}^{n}$ such that $X_{i} \in[0,1]$ for every $i$ and $\mathbf{E}(X)=\mu I$ with $\mu \in[0,1]$. Let $\varepsilon \in(0,1 / 2)$. Then

$$
\mathbf{P}\left(\frac{1}{\mu T} \sum_{i=1}^{T} X_{i} \notin[1-\varepsilon, 1+\varepsilon]\right) \leq 2 n \cdot \exp \left(-T \frac{\varepsilon^{2} \mu}{2 \ln 2}\right) .
$$

In our case, $\mathbf{E}(X)=(1 / n) I$ and $X \in[0,1]$. So $\mu=1 / n$. Thus, if $T>(2 \ln 2) \cdot \frac{\ln n+2 \ln 2}{\varepsilon^{2} \mu}=$ $O\left(n \log n / \varepsilon^{2}\right)$, then $\mathbf{P}\left(\frac{1}{\mu T} \sum_{i=1}^{T} X_{i} \notin[1-\varepsilon, 1+\varepsilon]\right)<1 / 2$. Thus, with constant probability, we obtain a solution $y$ to Problem 2 where $y$ has only $O\left(n \log n / \varepsilon^{2}\right)$ non-zero entries.

\section{Solving Problem 2 by BSS}

In our modification of the BSS algorithm [4], we keep a matrix $A$ of the form $A=\sum_{i} y_{i} B_{i}$ with $y \geq 0$, starting with $A=0$, and at each iteration we add another term $\alpha B_{j}$ to $A$. We enforce the invariant that the eigenvalues of $A$ lie in $[\ell, u]$, where $u$ and $\ell$ are parameters given by $u=u_{0}+t \delta_{U}$ and $\ell=\ell_{0}+t \delta_{L}$ after $t$ iterations. This procedure is presented in Algorithm 1 . The step of the algorithm which finds $B_{j}$ and $\alpha$ can be done by exhaustive search on $j$ and binary search on $\alpha$. Instead of the binary search, one could also compare the quantities $U_{A(t-1)}\left(B_{j}\right)$ and $L_{A(t-1)}\left(B_{j}\right)$ defined below.

In the original BSS algorithm, the matrices are rank one: $B_{j}=v_{j} v_{j}^{T}$ for some vector $v_{j}$. Their Lemmas 3.3 and 3.4 give sufficient conditions on the new term $\alpha v_{j} v_{j}^{T}$ so that the invariant on the eigenvalues is maintained; Lemma 3.5 gives sufficient conditions on the remaining parameters so that a suitable new term $\alpha v_{j} v_{j}^{T}$ exists with $\alpha>0$. In this section we generalize those lemmas to allow $B_{i}$ matrices of arbitrary rank.

Let $A \in \mathbb{S}^{n}$. If $u \in \mathbb{R}$ with $\lambda_{\max }(A)<u$, define $\Phi^{u}(A):=\operatorname{Tr}(u I-A)^{-1}$. If $\ell \in \mathbb{R}$ with $\lambda_{\min }(A)>\ell$, define $\Phi_{\ell}(A):=\operatorname{Tr}(A-\ell I)^{-1}$. Note that $\Phi_{\ell}(A)=\sum_{i} 1 /\left(\lambda_{i}-\ell\right)$ and $\Phi^{u}(A)=\sum_{i} 1 /\left(u-\lambda_{i}\right)$, where $\lambda_{1}, \ldots, \lambda_{n}$ are the eigenvalues of $A$.

Lemma 19 (Analog of Lemma 3.3 in [4]). Let $A \in \mathbb{S}^{n}$ and $X \in \mathbb{S}_{+}^{n}$ with $X \neq 0$. Let $u \in \mathbb{R}$ and $\delta_{U}>0$. Suppose $\lambda_{\max }(A)<u$. Let $u^{\prime}:=u+\delta_{U}$ and $M:=u^{\prime} I-A$. If

$$
\frac{1}{\alpha} \geq \frac{\left\langle M^{-2}, X\right\rangle}{\Phi^{u}(A)-\Phi^{u^{\prime}}(A)}+\left\langle M^{-1}, X\right\rangle=: U_{A}(X),
$$

then $\lambda_{\max }(A+\alpha X)<u^{\prime}$ and $\Phi^{u^{\prime}}(A+\alpha X) \leq \Phi^{u}(A)$. 


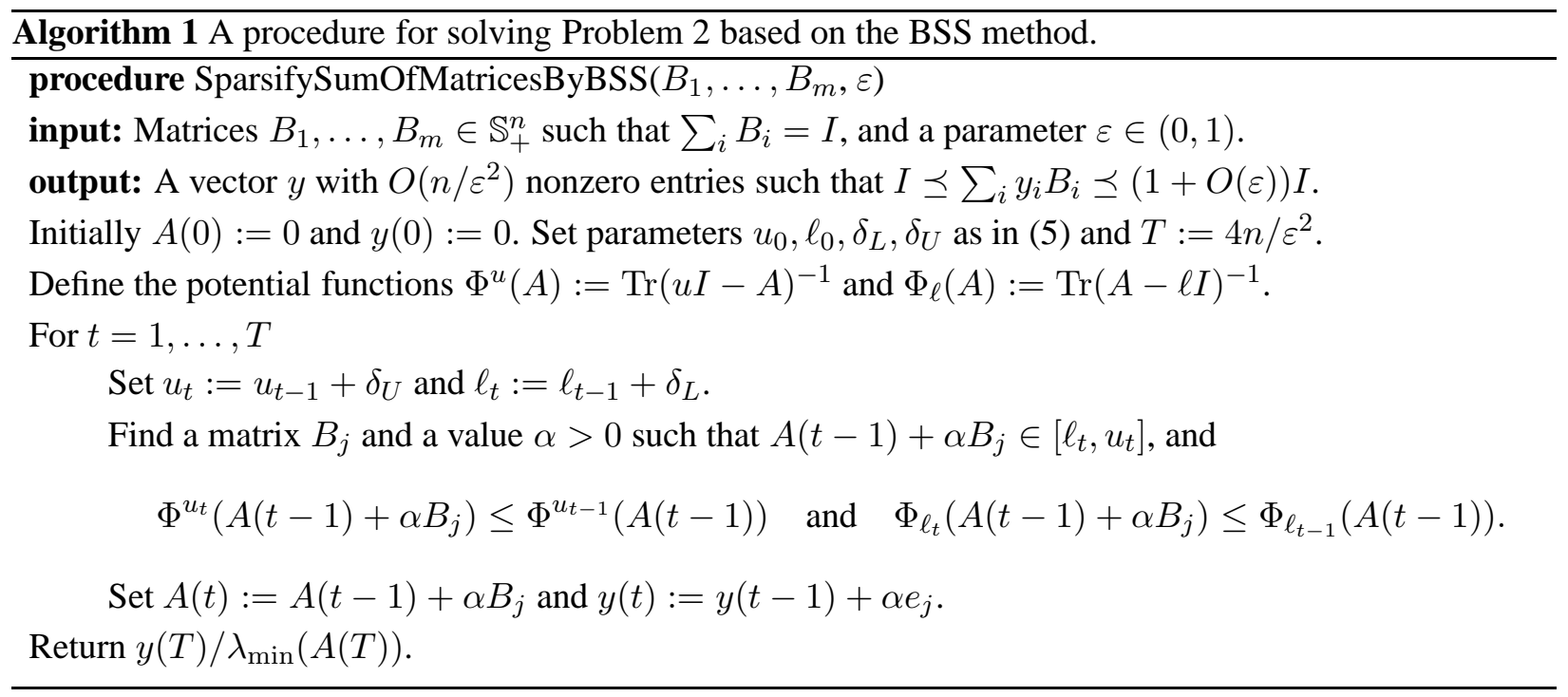

Proof. Clearly $M \succ 0$. Let $V:=X^{1 / 2}$. By the Sherman-Morrison-Woodbury formula [13],

$$
\begin{aligned}
\Phi^{u^{\prime}}(A+\alpha X) & =\operatorname{Tr}\left(M-\alpha V V^{T}\right)^{-1}=\operatorname{Tr}\left(M^{-1}+\alpha M^{-1} V\left(I-\alpha V^{T} M^{-1} V\right)^{-1} V^{T} M^{-1}\right) \\
& =\Phi^{u^{\prime}}(A)+\operatorname{Tr}\left(\alpha M^{-1} V\left(I-\alpha V^{T} M^{-1} V\right)^{-1} V^{T} M^{-1}\right) .
\end{aligned}
$$

Since $M^{-1} \succ 0, X \neq 0$ and $\Phi^{u}(A)>\Phi^{u^{\prime}}(A)$, our hypotheses imply $1 / \alpha>\left\langle M^{-1}, X\right\rangle=\operatorname{Tr}\left(V^{T} M^{-1} V\right) \geq$ $\lambda_{\max }\left(V^{T} M^{-1} V\right) \geq 0$, so $\beta:=\lambda_{\min }\left(I-\alpha V^{T} M^{-1} V\right)=1-\alpha \lambda_{\max }\left(V^{T} M^{-1} V\right)>0$ and by, e.g., [18, Corollary 7.7.4],

$$
0 \prec \beta I \preceq I-\alpha V^{T} M^{-1} V \Longrightarrow 0 \prec\left(I-\alpha V^{T} M^{-1} V\right)^{-1} \preceq \beta^{-1} I .
$$

Thus,

$$
\begin{aligned}
\Phi^{u^{\prime}}(A+\alpha X) & \leq \Phi^{u^{\prime}}(A)+\alpha \beta^{-1} \operatorname{Tr}\left(V^{T} M^{-2} V\right) \\
& =\Phi^{u}(A)-\left(\Phi^{u}(A)-\Phi^{u^{\prime}}(A)\right)+\alpha \beta^{-1}\left\langle M^{-2}, X\right\rangle
\end{aligned}
$$

To prove that $\Phi^{u^{\prime}}(A+\alpha X) \leq \Phi^{u}(A)$, it suffices to show that $\alpha \beta^{-1}\left\langle M^{-2}, X\right\rangle \leq \Phi^{u}(A)-\Phi^{u^{\prime}}(A)$. This is equivalent to

$$
\frac{\left\langle M^{-2}, X\right\rangle}{1 / \alpha-\lambda_{\max }\left(V^{T} M^{-1} V\right)} \leq \Phi^{u}(A)-\Phi^{u^{\prime}}(A)
$$

which follows from $1 / \alpha \geq U_{A}(X)$ since $\lambda_{\max }\left(V^{T} M^{-1} V\right) \leq \operatorname{Tr}\left(V^{T} M^{-1} V\right)=\left\langle M^{-1}, X\right\rangle$.

It remains to show that $\lambda_{\max }(A+\alpha X)<u^{\prime}$. Suppose not. Choose $\varepsilon \in\left(0, \delta_{U}\right)$ such that $1 / \varepsilon>\Phi^{u}(A)$. By continuity, for some $\alpha^{\prime} \in(0, \alpha)$ we have $\lambda_{\max }\left(A+\alpha^{\prime} X\right)=u^{\prime}-\varepsilon$. Since $1 / \alpha^{\prime} \geq 1 / \alpha \geq U_{A}(X)$, we get $\Phi^{u^{\prime}}\left(A+\alpha^{\prime} X\right) \geq 1 / \varepsilon>\Phi^{u}(A) \geq \Phi^{u^{\prime}}\left(A+\alpha^{\prime} X\right)$, a contradiction.

Lemma 20 (Analog of Lemma 3.4 in [4]). Let $A \in \mathbb{S}^{n}$ and $X \in \mathbb{S}_{+}^{n}$, with $n \geq 2$. Let $\ell \in \mathbb{R}$ and $\delta_{L}>0$. Suppose $\lambda_{\min }(A)>\ell$ and $\Phi_{\ell}(A) \leq 1 / \delta_{L}$. Let $\ell^{\prime}:=\ell+\delta_{L}$ and $N:=A-\ell^{\prime}$ I. If

$$
0<\frac{1}{\alpha} \leq \frac{\left\langle N^{-2}, X\right\rangle}{\Phi_{\ell^{\prime}}(A)-\Phi_{\ell}(A)}-\left\langle N^{-1}, X\right\rangle=: L_{A}(X)
$$

then $\lambda_{\min }(A+\alpha X)>\ell^{\prime}$ and $\Phi_{\ell^{\prime}}(A+\alpha X) \leq \Phi_{\ell}(A)$. Moreover, $N \succ 0$. 
Proof. Note that $\lambda_{\min }(A)>\ell$ and $\Phi_{\ell}(A) \leq 1 / \delta_{L}$ imply that $N \succ 0$, and therefore $\lambda_{\min }(A+\alpha X)>\ell^{\prime}$. Let $V:=X^{1 / 2}$. By the Sherman-Morrison-Woodbury formula,

$$
\begin{aligned}
\Phi_{\ell^{\prime}}(A+\alpha X) & =\operatorname{Tr}\left(N+\alpha V V^{T}\right)^{-1}=\operatorname{Tr}\left(N^{-1}-\alpha N^{-1} V\left(I+\alpha V^{T} N^{-1} V\right)^{-1} V^{T} N^{-1}\right) \\
& =\Phi_{\ell^{\prime}}(A)-\operatorname{Tr}\left(\alpha N^{-1} V\left(I+\alpha V^{T} N^{-1} V\right)^{-1} V^{T} N^{-1}\right) .
\end{aligned}
$$

For $\beta:=\lambda_{\max }\left(I+\alpha V^{T} N^{-1} V\right)$, we have

$$
0 \prec I+\alpha V^{T} N^{-1} V \preceq \beta I \Longrightarrow 0 \prec \beta^{-1} I \preceq\left(I+\alpha V^{T} N^{-1} V\right)^{-1} .
$$

Thus,

$$
\begin{aligned}
\Phi_{\ell^{\prime}}(A+\alpha X) & \leq \Phi_{\ell^{\prime}}(A)-\alpha \beta^{-1} \operatorname{Tr}\left(V^{T} N^{-2} V\right) \\
& =\Phi_{\ell}(A)+\left(\Phi_{\ell^{\prime}}(A)-\Phi_{\ell}(A)\right)-\alpha \beta^{-1}\left\langle N^{-2}, X\right\rangle
\end{aligned}
$$

We will be done if we show that $\alpha \beta^{-1}\left\langle N^{-2}, X\right\rangle \geq \Phi_{\ell^{\prime}}(A)-\Phi_{\ell}(A)$. This is equivalent to

$$
\frac{\left\langle N^{-2}, X\right\rangle}{1 / \alpha+\lambda_{\max }\left(V^{T} N^{-1} V\right)} \geq \Phi_{\ell^{\prime}}(A)-\Phi_{\ell}(A)
$$

which follows from $0<1 / \alpha \leq L_{A}(X)$, since $\Phi_{\ell^{\prime}}(A)>\Phi_{\ell}(A), N \succ 0$, and $\lambda_{\max }\left(V^{T} N^{-1} V\right) \leq$ $\operatorname{Tr}\left(V^{T} N^{-1} V\right)=\left\langle N^{-1}, X\right\rangle$.

The next lemma can be proved by a syntactic modification of the proof of Lemma 3.5 in [4].

Lemma 21 (Analog of Lemma 3.5 in [4]). Let $A \in \mathbb{S}^{n}$ with $n \geq 2$, and let $u, \ell \in \mathbb{R}$ and $\varepsilon_{U}, \delta_{U}, \varepsilon_{L}, \delta_{L}>0$ such that $\lambda_{\max }(A)<u, \lambda_{\min }(A)>\ell, \Phi^{u}(A) \leq \varepsilon_{U}$, and $\Phi_{\ell}(A) \leq \varepsilon_{L}$. Let $B_{1}, \ldots, B_{m} \in \mathbb{S}^{n}$ such that $\sum_{i} B_{i}=$ I. If

$$
0 \leq \frac{1}{\delta_{U}}+\varepsilon_{U} \leq \frac{1}{\delta_{L}}-\varepsilon_{L}
$$

then there exists $j \in[m]$ and $\alpha>0$ for which $L_{A}\left(B_{j}\right) \geq 1 / \alpha \geq U_{A}\left(B_{j}\right)$.

Proof. As in [4, Lemma 3.5], it suffices to show that $\sum_{i} L_{A}\left(B_{i}\right) \geq \sum_{i} U_{A}\left(B_{i}\right)$. Let $u^{\prime}:=u+\delta_{U}$, $M:=u^{\prime} I-A, \ell^{\prime}:=\ell+\delta_{L}$, and $N:=A-\ell^{\prime} I$. It follows from the bilinearity of $\langle\cdot, \cdot\rangle$ and the assumption $\sum_{i} B_{i}=I$ that

$$
\begin{gathered}
\sum_{i} U_{A}\left(B_{i}\right)=\frac{\operatorname{Tr} M^{-2}}{\Phi^{u}(A)-\Phi^{u^{\prime}}(A)}+\operatorname{Tr} M^{-1} \\
\sum_{i} L_{A}\left(B_{i}\right)=\frac{\operatorname{Tr} N^{-2}}{\Phi_{\ell^{\prime}}(A)-\Phi_{\ell}(A)}-\operatorname{Tr} N^{-1}
\end{gathered}
$$

It is shown in [4, Lemma 3.5] that (4a) is at most (4b), completing the proof.

Now we set the parameters of Lemma 21] similarly as in [4]:

$$
\delta_{L}:=1 \quad \varepsilon_{L}:=\frac{\varepsilon}{2} \quad \ell_{0}:=-\frac{n}{\varepsilon_{L}} \quad \delta_{U}:=\frac{2+\varepsilon}{2-\varepsilon} \quad \varepsilon_{U}:=\frac{\varepsilon}{2 \delta_{U}} \quad u_{0}:=\frac{n}{\varepsilon_{U}} .
$$

So (3) holds with equality. If $A$ is the matrix obtained after $T=4 n / \varepsilon^{2}$ iterations, then

$$
\frac{\lambda_{\max }(A)}{\lambda_{\min }(A)} \leq \frac{u_{0}+T \delta_{U}}{\ell_{0}+T \delta_{L}}=\left(\frac{2+\varepsilon}{2-\varepsilon}\right)^{2} \leq \frac{1+\varepsilon}{1-\varepsilon}
$$

so $A^{\prime}:=A / \lambda_{\min }(A)$ satisfies $I \preceq A^{\prime} \preceq(1+\varepsilon) I /(1-\varepsilon)$ and $A^{\prime}$ is a positive linear combination of $O\left(n / \varepsilon^{2}\right)$ of the matrices $B_{i}$.

It is easy to check that the previous lemmas also hold if we replace the set $\mathbb{S}^{n}$ of symmetric matrices of size $n \times n$ by the set $\mathbb{H}^{n}$ of Hermitian matrices of size $n \times n$. 


\subsection{Running Time}

At each iteration, we must compute $U_{A}\left(B_{j}\right)$ and $L_{A}\left(B_{j}\right)$ for each $j \in[m]$. The functions $U_{A}(X)$ and $L_{A}(X)$ are the inner products of $X$ with certain matrices that can be obtained from $A$ in time $O\left(n^{3}\right)$. Thus, each iteration runs in time $O\left(n^{3}+m n^{2}\right)=O\left(m n^{2}\right)$, and the total running time after $T=4 n / \varepsilon^{2}$ iterations is $O\left(m n^{3} / \varepsilon^{2}\right)$. We remark that the reduction to the case $B=I$ can be made in time $O\left(m n^{3}\right)$. This concludes the proof of Theorem 3

If the matrices $B_{i}$ have $O(1)$ nonzero entries, as in the graph sparsification problem, the algorithm can be made to run in time $O\left(n^{4} / \varepsilon^{2}+m n / \varepsilon^{2}\right)$. We briefly sketch the details. To reduce the problem to the case that $B=I$, we first compute $\left(B^{+}\right)^{1 / 2}$, where $B^{+}$is the Moore-Penrose pseudoinverse of $B$. Define the function $f(X):=\left(B^{+}\right)^{1 / 2} X\left(B^{+}\right)^{1 / 2}$ on $\mathbb{S}^{n}$.

The reduction now calls for replacing each input matrix $B_{i}$ by $f\left(B_{i}\right)$ and the matrix $B$ by $f(B)$. But we shall not do this. Instead, we do some preprocessing at each iteration as follows. The function $U_{A}(X)$ (as well as $\left.L_{A}(X)\right)$ is the inner product of $X$ with a certain matrix $V$. Hence, $U_{A}\left(f\left(B_{j}\right)\right)=\left\langle V, f\left(B_{j}\right)\right\rangle=$ $\left\langle f(V), B_{j}\right\rangle$ for every $j$, since $f$ is self-adjoint. Thus, to compute $U_{A}\left(f\left(B_{j}\right)\right)$ for each $j$, we first compute the matrix $f(V)$ in time $O\left(n^{3}\right)$, and now the inner product $U_{A}\left(f\left(B_{j}\right)\right)=\left\langle f(V), B_{j}\right\rangle$ can be computed in constant time for each $j$, since $B_{j}$ has $O(1)$ nonzero entries. Thus, each iteration runs in time $O\left(n^{3}+m\right)$ and the total running time is $O\left(n^{4} / \varepsilon^{2}+m n / \varepsilon^{2}\right)$.

\section{Solving Problem 2 by MMWUM}

Observe that the set of all vectors $y$ that are feasible for (2) is the feasible region of a semidefinite program (SDP). So solving Problem 2 amounts to finding a sparse solution to this SDP. Here "sparse" means that there are few non-zero entries in the solution $y$; this differs from other notions of "low-complexity" SDP solutions, such as the low-rank solutions studied by So, Ye and Zhang [36].

It has long been known known that the multiplicative weight update method can be used to construct sparse solutions for some linear programs. A prominent example is the construction of sparse, low-regret solutions to zero-sum games [9, 43, 44]. (Another example is the work of Charikar et al. [7] on approximating metrics by few tree metrics.) Building on that idea, one might imagine that Arora and Kale's matrix multiplicative update method (MMWUM) [3] can construct sparse solutions to (2). In this section, we show that this is indeed possible: we obtain a solution $y$ to Problem 2 with $O\left(n \log n / \varepsilon^{3}\right)$ nonzero entries.

\subsection{Overview of MMWUM}

The MMWUM is an algorithm that helps us approximately solve an SDP feasibility problem. The gist of (a slight modification of) the method is contained in the following result (its proof can be found in Appendix $B$ ):

Theorem 22. Let $T, K, n_{1}, \ldots, n_{K}$ be positive integers. Let $C_{k}, A_{1, k}, \ldots, A_{m, k} \in \mathbb{S}^{n} k$ for $k \in[K]$. For each $k \in[K]$, let $\eta_{k}>0$ and $0<\beta_{k} \leq 1 / 2$. Given $X_{1}, \ldots, X_{K} \in \mathbb{S}^{n}$, consider the system

$$
\sum_{i=1}^{m} y_{i}\left\langle A_{i, k}, X_{k}\right\rangle \geq\left\langle C_{k}, X_{k}\right\rangle-\eta_{k} \operatorname{Tr} X_{k}, \quad \forall k \in[K], \quad \text { and } \quad y \in \mathbb{R}_{+}^{m} .
$$

For each $k \in[K]$, let $\left\{\mathcal{P}_{k}, \mathcal{N}_{k}\right\}$ be a partition of $[T]$, let $0<\ell_{k} \leq \rho_{k}$, and let $W_{k}^{(t)} \in \mathbb{S}^{n}$ and $\ell_{k}^{(t)} \in \mathbb{R}$ for $t \in[T+1]$. Let $y^{(t)} \in \mathbb{R}^{m}$ for $t \in[T]$. Suppose the following properties hold:

$$
W_{k}^{(t+1)}=\exp \left(-\frac{\beta_{k}}{\ell_{k}+\rho_{k}} \sum_{\tau=1}^{t}\left[\sum_{i=1}^{m} y_{i}^{(\tau)} A_{i, k}-C_{k}+\ell_{k}^{(\tau)} I\right]\right), \quad \forall t \in\{0, \ldots, T\}, \forall k \in[K],
$$




$$
\begin{gathered}
y=y^{(t)} \text { is a solution for (6) with } X_{k}=W_{k}^{(t)}, \forall k \in[K], \quad \forall t \in[T], \\
\sum_{i=1}^{m} y_{i}^{(t)} A_{i, k}-C_{k} \in\left\{\begin{array}{l}
{\left[-\ell_{k}, \rho_{k}\right], \quad \text { if } t \in \mathcal{P}_{k},} \\
{\left[-\rho_{k}, \ell_{k}\right], \quad \text { if } t \in \mathcal{N}_{k},}
\end{array} \quad \forall t \in[T], k \in[K],\right. \\
\ell_{k}^{(t)}=\ell_{k}, \quad \forall t \in \mathcal{P}_{k}, \forall k \in[K], \quad \text { and } \quad \ell_{k}^{(t)}=-\ell_{k}, \quad \forall t \in \mathcal{N}_{k}, \forall k \in[K] .
\end{gathered}
$$

Define $\bar{y}:=\frac{1}{T} \sum_{t=1}^{T} y^{(t)}$. Then,

$$
\sum_{i=1}^{m} \bar{y}_{i} A_{i, k}-C_{k} \succeq-\left[\beta_{k} \ell_{k}+\frac{\left(\rho_{k}+\ell_{k}\right) \ln n}{T \beta_{k}}+\left(1+\beta_{k}\right) \eta_{k}\right] I, \quad \forall k \in[K] .
$$

Take $K=2$, set $C_{1}:=I$ and $C_{2}:=-I$, and put $A_{i, 1}:=B_{i}$ and $A_{i, 2}:=-B_{i}$ for each $i \in[m]$. Then Theorem 22 shows that finding a solution for (2) reduces to constructing an oracle that solves linear systems of the form (6) with a few extra technical properties involving the parameters $\ell_{k}$ and $\rho_{k}$, and adjusting the other parameters so that the error term on the right-hand side of (7) is $\leq \varepsilon$.

To obtain a feasible solution for (2) that is also sparse, the idea is to design an implementation of the oracle that returns a vector $y^{(t)}$ with only one nonzero entry at each iteration $t$ of MMWUM, and to adjust the parameters so that, after $T=O\left(n \log n / \varepsilon^{3}\right)$ iterations, the smallest and largest eigenvalues of $\sum_{i=1}^{m} \bar{y}_{i} B_{i}$ are $\varepsilon$-close to 1 . Since $\bar{y}$ is the average of the $y^{(t)}$ 's, the resulting $\bar{y}$ will have at most $T$ nonzero entries.

We set the remaining parameters as follows:

$$
\begin{gathered}
\beta:=\beta_{1}:=\beta_{2}:=\frac{\varepsilon}{4}, \quad T:=\frac{2(\rho+\ell) \ln n}{\beta \varepsilon}, \quad \eta:=\eta_{1}:=\eta_{2}:=\frac{\varepsilon}{8}, \\
\ell:=\ell_{1}:=\ell_{2}:=1, \quad \rho:=\rho_{1}:=\rho_{2}:=\frac{1+\eta}{\eta} n, \quad \mathcal{P}_{1}:=\mathcal{N}_{2}:=[T], \quad \mathcal{N}_{1}:=\mathcal{P}_{2}:=\emptyset .
\end{gathered}
$$

Then the error term on the right-hand side of (7) is

$$
\beta \ell+\frac{(\rho+\ell) \ln n}{T \beta}+(1+\beta) \eta=\frac{\varepsilon}{4}+\frac{\varepsilon}{2}+\left(1+\frac{\varepsilon}{4}\right) \frac{\varepsilon}{8}=\frac{7 \varepsilon}{8}+\frac{\varepsilon^{2}}{32} \leq \varepsilon .
$$

Thus, (2) follows from (7) and (8). Moreover, $T=O\left(n \log n / \varepsilon^{3}\right)$, as desired.

\subsection{The Oracle}

It remains to implement the oracle. Consider an iteration $t$, and let $X_{1}:=W_{1}^{(t)}$ and $X_{2}:=W_{2}^{(t)}$ be given. We must find $y:=y^{(t)} \in \mathbb{R}_{+}^{m}$ with at most one nonzero entry such that

$$
\sum_{i=1}^{m} y_{i}\left\langle X_{1}, B_{i}\right\rangle \geq(1-\eta) \operatorname{Tr} X_{1}, \quad \sum_{i=1}^{m} y_{i}\left\langle X_{2}, B_{i}\right\rangle \leq(1+\eta) \operatorname{Tr} X_{2}, \quad \text { and } \quad \sum_{i=1}^{m} y_{i} B_{i} \in[0, \rho] .
$$

Since $y$ should have only one nonzero entry, it suffices to find $j \in[m]$ and $\alpha \in \mathbb{R}_{+}$such that

$$
\begin{aligned}
\alpha\left\langle X_{1}, B_{j}\right\rangle & \geq(1-\eta) \operatorname{Tr} X_{1}, \\
\alpha\left\langle X_{2}, B_{j}\right\rangle & \leq(1+\eta) \operatorname{Tr} X_{2}, \\
\alpha \operatorname{Tr} B_{j} & \leq \rho .
\end{aligned}
$$

Here we are using the fact that $\lambda_{\max }\left(B_{j}\right) \leq \operatorname{Tr} B_{j}$ since $B_{j} \succeq 0$. We will show that such $j$ and $\alpha$ exist. Due to the definition of $W_{1}$ and $W_{2}$, the oracle can assume that $X_{1}$ is a scalar multiple of $X_{2}^{-1}$, although we will not make use of that fact. 
Proposition 23. Let $B_{1}, \ldots, B_{m} \in \mathbb{S}_{+}^{n}$ such that $\sum_{i=1}^{m} B_{i}=I$. Let $\eta>0$ and $X_{1}, X_{2} \in \mathbb{S}_{++}^{n}$. Then, for $\rho:=(1+\eta) n / \eta$, there exist $j \in[m]$ and $\alpha \geq 0$ such that (9) holds.

Proof. By possibly dropping some $B_{i}$ 's, we may assume that $B_{i} \neq 0$ for every $i \in[m]$. Define $p_{i}:=$ $\left\langle X_{1}, B_{i}\right\rangle / \operatorname{Tr} X_{1}>0$ for every $i \in[m]$. Consider the probability space on $[m]$ where $j$ is sampled from $[m]$ with probability $p_{j}$. The fact that $\sum_{j=1}^{m} p_{j}=1$ follows from $\sum_{i=1}^{m} B_{i}=I$. Then $\mathbf{E}_{j}\left[p_{j}^{-1} \operatorname{Tr} B_{j}\right]=$ $\sum_{i=1}^{m} \operatorname{Tr} B_{i}=\operatorname{Tr} I=n$. By Markov's inequality,

$$
\mathbf{P}\left(p_{j}^{-1} \operatorname{Tr} B_{j} \leq \frac{(1+\eta)}{\eta} n\right)=1-\mathbf{P}\left(p_{j}^{-1} \operatorname{Tr} B_{j}>\frac{(1+\eta)}{\eta} n\right)>1-\frac{\eta}{1+\eta}=\frac{1}{1+\eta} .
$$

Next note that $\mathbf{E}_{j}\left[p_{j}^{-1}\left\langle X_{2}, B_{j}\right\rangle\right]=\sum_{i=1}^{m}\left\langle X_{2}, B_{i}\right\rangle=\left\langle X_{2}, I\right\rangle=\operatorname{Tr} X_{2}$. Together with Markov's inequality, this yields

$$
\mathbf{P}\left(p_{j}^{-1}\left\langle X_{2}, B_{j}\right\rangle \leq(1+\eta) \operatorname{Tr} X_{2}\right)=1-\mathbf{P}\left(p_{j}^{-1}\left\langle X_{2}, B_{j}\right\rangle>(1+\eta) \operatorname{Tr} X_{2}\right)>1-\frac{1}{1+\eta} .
$$

It follows from (10) and (11) that there exists $j \in[m]$ satisfying

$$
p_{j}^{-1}\left\langle X_{2}, B_{j}\right\rangle \leq(1+\eta) \operatorname{Tr} X_{2}, \quad \text { and } \quad p_{j}^{-1} \operatorname{Tr} B_{j} \leq \frac{1+\eta}{\eta} n=\rho .
$$

Set $\alpha:=p_{j}^{-1}$ and note that

$$
\alpha\left\langle X_{1}, B_{j}\right\rangle=p_{j}^{-1}\left\langle X_{1}, B_{j}\right\rangle=\operatorname{Tr} X_{1} \geq(1-\eta) \operatorname{Tr} X_{1} .
$$

Hence, $j$ and $\alpha$ satisfy (9).

The following proposition, proven in Appendix C] shows that the parameters achieved by Proposition 23 is essentially optimal.

Proposition 24. Any oracle for satisfying (9) must have $\rho=\Omega(n / \eta)$, even if the $B_{i}$ matrices have rank one, and even if $X_{1}$ is a scalar multiple of $X_{2}^{-1}$.

We also point out that a naive application of MMWUM as stated by Kale in [22] does not work. In his description of MMWUM, the parameter $K$ is fixed as 1 . So we must correspondingly adjust our input matrices to be block-diagonal, e.g., $C$ has two blocks: $I$ and $-I$. However, applying Theorem 22 in this manner would lead to a sparsifier with $\Omega\left(n^{2}\right)$ edges. The reason is that the parameter $\rho$ needs to be $\Omega(n)$, and we must choose $\ell=\rho$ since the spectrum of $\sum_{i=1}^{m} y_{i} A_{i}-C$ is symmetric around zero for any $y$. Thus, to get the error term on the right-hand side of (7) to be $\leq \varepsilon$, we would need to take $T=\Omega\left(n^{2}\right)$.

\section{Solving Problem 2 by a Width-Free MMWUM}

The algorithm of Section 5 solves Problem 2 with only $O\left(n \log n / \varepsilon^{3}\right)$ nonzero entries, which is slightly worse than the $O\left(n \log n / \varepsilon^{2}\right)$ nonzero entries achieved by the Ahlswede-Winter method discussed in Section 3. The main reason for this discrepancy is that MMWUM requires us to bound the "width" of the oracle using the parameter $\rho$; formally, the oracle must the inequality $\alpha \operatorname{Tr} B_{j} \leq \rho$ in (9). In order to satisfy this width constraint, the oracle loses an extra factor of $O(1 / \varepsilon)$, and this is necessary as shown in Proposition 24

In this section, we slightly refine MMWUM to avoid its dependence on the width. This allows us to simplify our oracle and avoid losing the extra factor of $O(1 / \varepsilon)$. We obtain a solution to Problem 2 with only 
only $O\left(n \log n / \varepsilon^{2}\right)$ nonzero entries, matching the sparsity of the solutions obtained by the Ahlswede-Winter inequality.

The following theorem is our width-free variant of MMWUM. We remark that the method described in this theorem is geared towards solving Problem 2 and is not necessarily useful for all applications of MMWUM.

Theorem 25. Let $T$ be a positive integer. Let $B_{1}, \ldots, B_{m} \in \mathbb{S}_{+}^{n}$ be nonzero. Let $\gamma, \eta, \delta_{L}, \delta_{U}>0$. For any given $X_{L}, X_{U} \in \mathbb{S}^{n}$, consider the system

$$
\begin{aligned}
& \delta_{U} \geq \frac{\exp \left(\gamma \alpha \operatorname{Tr} B_{j}\right)-1}{\operatorname{Tr} B_{j}}\left\langle X_{U}, B_{j}\right\rangle, \\
& \delta_{L} \leq \frac{1-\exp \left(-\gamma \alpha \operatorname{Tr} B_{j}\right)}{\operatorname{Tr} B_{j}}\left\langle X_{L}, B_{j}\right\rangle, \\
& \alpha \in \mathbb{R}_{+}, \quad j \in[m] .
\end{aligned}
$$

For each $t \in\{0, \ldots, T+1\}$, let $A(t), W_{L}(t), W_{U}(t) \in \mathbb{S}^{n}$, let $\alpha(t) \in \mathbb{R}_{+}$, and let $j(t) \in[m]$. Suppose the following properties hold:

$$
A(t)=\sum_{\tau=1}^{t} \alpha(\tau) B_{j(\tau)}, \quad \forall t \in\{0, \ldots, T\},
$$

$$
W_{U}(t+1)=\exp (\gamma A(t)) \quad \text { and } \quad W_{L}(t+1)=\exp (-\gamma A(t)), \quad \forall t \in\{0, \ldots, T\},
$$

$\left(\alpha, B_{j}\right)=\left(\alpha(t), B_{j(t)}\right)$ is a solution for (12) with $\left(X_{U}, X_{L}\right)=\left(\frac{W_{U}(t)}{\operatorname{Tr} W_{U}(t)}, \frac{W_{L}(t)}{\operatorname{Tr} W_{L}(t)}\right), \quad \forall t \in[T]$.

Then

$$
\frac{A(T)}{T} \in\left[\frac{\log \left(1-\delta_{L}\right)^{-1}}{\gamma}-\frac{\log n}{T \gamma}, \frac{\log \left(1+\delta_{U}\right)}{\gamma}+\frac{\log n}{T \gamma}\right]
$$

Proof. We will use Golden-Thompson inequality:

$$
\operatorname{Tr}(\exp (A+B)) \leq \operatorname{Tr}(\exp (A) \exp (B)), \quad \forall A, B \in \mathbb{S}^{n} .
$$

We will also make use of the following facts. First,

$$
\exp (c x) \leq 1+\frac{\exp (c \cdot b)-1}{b} x \quad \forall c \in \mathbb{R}, b>0, x \in[0, b] .
$$

For $X \in \mathbb{S}_{+}^{n}$, we have $\lambda_{\max }(X) \leq \operatorname{Tr} X$, so $X \in[0, \operatorname{Tr} X]$, and

$$
\exp (c X) \preceq I+\frac{\exp (c \cdot \operatorname{Tr} X)-1}{\operatorname{Tr} X} X .
$$

For each $t \in[T+1]$, define $\Phi_{L}(t):=\operatorname{Tr} W_{L}(t)$ and $\Phi_{U}(t)=\operatorname{Tr} W_{U}(t)$. For each $t \in[T]$,

$$
\begin{aligned}
\Phi_{U}(t+1) & =\operatorname{Tr}(\exp (\gamma A(t)))=\operatorname{Tr}\left(\exp \left(\gamma A(t-1)+\gamma \alpha B_{j}\right)\right) \\
& \stackrel{\text { 114) }}{\leq} \operatorname{Tr}\left(\exp (\gamma A(t-1)) \exp \left(\gamma \alpha B_{j}\right)\right) \\
& \stackrel{[15]}{\leq} \operatorname{Tr}\left(\exp (\gamma A(t-1))\left(\frac{\exp \left(\gamma \alpha \operatorname{Tr} B_{j}\right)-1}{\operatorname{Tr} B_{j}} B_{j}+I\right)\right) \\
& =\frac{\exp \left(\gamma \alpha \operatorname{Tr} B_{j}\right)-1}{\operatorname{Tr} B_{j}} \operatorname{Tr}\left(\exp (\gamma A(t-1)) B_{j}\right)+\operatorname{Tr}(\exp (\gamma A(t-1))) \\
& =\frac{\exp \left(\gamma \alpha \operatorname{Tr} B_{j}\right)-1}{\operatorname{Tr} B_{j}}\left\langle W_{U}(t), B_{j}\right\rangle+\Phi_{U}(t) \\
& \stackrel{112}{\leq}\left(1+\delta_{U}\right) \Phi_{U}(t),
\end{aligned}
$$


where we abbreviated $j:=j(t)$ and $\alpha:=\alpha(t)$.

Since $A(0)=0$, we have that $\Phi_{U}(1)=\operatorname{Tr} I=n$. Using (16), after $T$ iterations,

$$
\Phi_{U}(T+1) \leq\left(1+\delta_{U}\right)^{T} n
$$

Thus,

$$
\exp \left(\gamma \lambda_{\max }(A(T))\right) \leq \sum_{i=1}^{n} \exp \left(\gamma \lambda_{i}\right)=\operatorname{Tr} W_{U}(T+1)=\Phi_{U}(T+1) \leq\left(1+\delta_{U}\right)^{T} n,
$$

where $\lambda_{1}, \ldots, \lambda_{n}$ are the eigenvalues of $A(T)$. And so $\gamma \lambda_{\max }(A(T)) \leq T \log \left(1+\delta_{U}\right)+\log n$, which implies the upper bound in (13). The proof of the lower bound is analogous.

Next we establish conditions under which we can construct an oracle for solving the system (12). The proof consists of algebraic manipulations and an averaging argument analogous to the proof of Lemma 3.5 in [4].

Theorem 26. Let $B_{1}, \ldots, B_{m} \in \mathbb{S}_{+}^{n}$ be nonzero such that $\sum_{i=1}^{m} B_{i}=I$. Let $\delta_{U}, \delta_{L}>0$ be such that

$$
\frac{1}{\delta_{L}}-n \geq \frac{1}{\delta_{U}}
$$

Then, for any $X_{L}, X_{U} \in \mathbb{S}_{++}^{n}$ with trace one, the system (12) has a solution.

Proof. The first inequality in (12) is equivalent to

$$
\frac{\operatorname{Tr} B_{j}}{\exp \left(\gamma \alpha \operatorname{Tr} B_{j}\right)-1} \geq \frac{\left\langle X_{U}, B_{j}\right\rangle}{\delta_{U}}
$$

Using the identity $\frac{1}{1-1 / x}=1+\frac{1}{x-1}$, the second inequality in (12) is equivalent to

$$
\frac{\operatorname{Tr} B_{j}}{\exp \left(\gamma \alpha \operatorname{Tr} B_{j}\right)-1} \leq \frac{\left\langle X_{L}, B_{j}\right\rangle}{\delta_{L}}-\operatorname{Tr} B_{j} .
$$

We will choose $j \in[m]$ so that

$$
\frac{\left\langle X_{L}, B_{j}\right\rangle}{\delta_{L}}-\operatorname{Tr} B_{j} \geq \frac{\left\langle X_{U}, B_{j}\right\rangle}{\delta_{U}}
$$

and set $\alpha$ so that (18) holds with equality. Then both (18) and (19) will hold. Note that $\alpha \geq 0$ since $e^{\gamma \alpha \operatorname{Tr} B_{j}}=1+\delta_{U} \operatorname{Tr} B_{j} /\left\langle X_{U}, B_{j}\right\rangle>1$ and $\gamma \operatorname{Tr} B_{j}>0$.

To see that there exists $j \in[m]$ satisfying (20), note that, by (17) and $\sum_{i=1}^{m} B_{i}=I$,

$$
\sum_{i=1}^{m}\left[\frac{\left\langle X_{L}, B_{i}\right\rangle}{\delta_{L}}-\operatorname{Tr} B_{i}\right]=\frac{\operatorname{Tr} X_{L}}{\delta_{L}}-n=\frac{1}{\delta_{L}}-n \geq \frac{1}{\delta_{U}}=\frac{\operatorname{Tr} X_{U}}{\delta_{U}}=\sum_{i=1}^{m} \frac{\left\langle X_{U}, B_{i}\right\rangle}{\delta_{U}}
$$

This concludes the proof.

Finally, let us show how to set the parameters to get a sparsifier. Given $\varepsilon \in(0,1)$, set

$$
\eta:=\varepsilon / 2, \quad \delta_{U}:=\frac{\eta}{n}, \quad \delta_{L}:=\frac{\eta}{(1+\eta) n}, \quad T:=\frac{n \log n}{\eta^{2}} .
$$


By our choice of $\delta_{L}$ and $\delta_{U}$, we have $1 / \delta_{L}-n=(1+\eta) n / \eta-n=n / \eta=1 / \delta_{U}$, so (17) holds with equality. After we run the modified version of MMWUM given by Theorem 25 , we obtain a matrix $A(T)$. Set $\bar{A}:=A(T) / T$. By Theorem 25 ,

$$
\lambda_{\max }(\bar{A}) \leq \frac{\log \left(1+\delta_{U}\right)}{\gamma}+\frac{\log n}{T \gamma} \leq\left(\delta_{U}+\frac{\eta^{2}}{n}\right) / \gamma=\frac{1+\eta}{n \gamma / \eta} .
$$

We will use that $-\log (1-x) \geq x$ for $x<1$. Thus,

$$
\lambda_{\min }(\bar{A}) \geq \frac{\log \left(1-\delta_{L}\right)^{-1}}{\gamma}-\frac{\log n}{T \gamma} \geq\left(\delta_{L}-\frac{\eta^{2}}{n}\right) / \gamma=\frac{1 /(1+\eta)-\eta}{n \gamma / \eta} \geq \frac{1-2 \eta}{n \gamma / \eta} .
$$

So if we choose $\gamma=\eta / n$ then $(1-\varepsilon) I \preceq \bar{A} \preceq(1+\varepsilon) I$ and $\bar{A}$ is of the form $\sum_{i} y_{i} B_{i}$ with $y \geq 0$ and has at most $T=O\left(n \log n / \varepsilon^{2}\right)$ nonzero entries.

Remark. The choice of $\gamma$ is actually irrelevant here. We could choose $\gamma>0$ arbitrarily, then define $\bar{A}=A(T) \cdot(n \gamma / \eta T)$ and the desired conclusion would hold.

\section{Solving Problem 2 by Pessimistic Estimators}

An anonymous reviewer for a preliminary draft of this paper raised the possibility of designing another deterministic solution to Problem 2. The proposal was to use the pessimistic estimators of Wigderson and Xiao [42] to derandomize the random sampling approach of Section 3. In this section we show that this proposal indeed works. We remark that pessimistic estimators were also used by Hofmeister and Lefmann [17] to derandomize the proof of Theorem 15 .

It is known that there is a close relationship between pessimistic estimators and multiplicative weight update methods. (See, for example, the work of Young [44].) However, the two methods are not identical, and in particular the algorithm presented in this section is not identical to either of our algorithms based on MMWUM. To illustrate one difference, notice that the algorithm in Section 3 has the property that its output vector $y$ has every component $y_{i}$ equal to an integer multiple of $n /\left(T \cdot \operatorname{Tr} B_{i}\right)$. The algorithm of this section also has that property as it is a derandomization of the algorithm in Section 3 . However, the algorithms in Sections 4, 5] and 6 do not have that property.

Definition 27 (Definition 3.1 in [42]). Let $\vec{X}=\left(X_{1}, \ldots, X_{T}\right)$ be random variables distributed over $[m]$. Let $S$ be an event with $\mathbf{P}(\vec{X} \in S)>0$. We say that $\phi_{0}, \ldots, \phi_{T}, \phi_{i}:[m]^{i} \rightarrow[0,1]$, are pessimistic estimators for $S$ if the following hold.

1. For any $i$ and any fixed $x_{1}, \ldots, x_{i} \in[m]$, we have that

$$
\mathbf{P}_{X_{i+1}, \ldots, X_{T}}\left(\left(x_{1}, \ldots, x_{i}, X_{i+1}, \ldots, X_{T}\right) \notin S\right) \leq \phi_{i}\left(x_{1}, \ldots, x_{i}\right) .
$$

2. For any $i$ and any fixed $x_{1}, \ldots, x_{i} \in[n]$ :

$$
\mathbf{E}_{X_{i+1}}\left(\phi_{i+1}\left(x_{1}, \ldots, x_{i}, X_{i+1}\right)\right) \leq \phi_{i}\left(x_{1}, \ldots, x_{i}\right) .
$$

Note that the function $\phi_{0}$ depends on no variables and is therefore just a scalar in $[0,1]$. A nice property of this definition is that it allows compositions very easily. That is, if we have pessimistic estimators $\phi_{0}, \ldots, \phi_{T}$ and $\psi_{0}, \ldots, \psi_{T}$ for events $S$ and $S^{\prime}$, resp., then $\phi_{0}+\psi_{0}, \ldots, \phi_{T}+\psi_{T}$ are pessimistic estimators for the event $S \cap S^{\prime}$ (see Lemma 3.3 in [42]). 
The key point of this method is that, if there are pessimistic estimators $\phi_{0}, \ldots, \phi_{T}$, such that $\phi_{0}<1$ and each $\phi_{i}$ can be computed efficiently, then one can find $\left(x_{1}, \ldots, x_{T}\right) \in S$ efficiently.

Let $X_{1}, \ldots, X_{T}$ be be i.i.d. random variables with same distribution as the random variable $X$ as defined in Section 3. Wigderson and Xiao [42] considered the event

$$
S_{\geq}=\left\{\vec{X}: \frac{1}{T} \sum_{i=1}^{T} X_{i} \succeq(1-\varepsilon) \mu I\right\}
$$

and obtained 1 the following pessimistic estimators:

$$
\begin{aligned}
& \phi_{0}=n e^{t T(1-\varepsilon) \mu}\left\|\mathbf{E}_{X}(\exp (-t X))\right\|^{T} \leq n \exp \left(-T \varepsilon^{2} \mu /(2 \ln 2)\right) \\
& \phi_{i}\left(x_{1}, \ldots, x_{i}\right):=e^{t T(1-\varepsilon) \mu} \operatorname{Tr}\left(\exp \left(-\sum_{i=1}^{j} t x_{i}\right)\right) \cdot\left\|\mathbf{E}_{X}(\exp (-t X))\right\|^{T-i}
\end{aligned}
$$

where $t=\log \left(\frac{1-(1-\varepsilon) \mu}{(1-\mu)(1-\varepsilon)}\right)$. Similarly, for the event $S_{\leq}=\left\{\vec{X}: \frac{1}{T} \sum_{i=1}^{T} X_{i} \preceq(1+\varepsilon) \mu I\right\}$, one can find the following pessimistic estimators

$$
\begin{aligned}
& \psi_{0}=n e^{-t^{\prime} T(1+\varepsilon) \mu}\left\|\mathbf{E}_{X}\left(\exp \left(t^{\prime} X\right)\right)\right\|^{T} \leq n \exp \left(-T \varepsilon^{2} \mu /(2 \ln 2)\right) \\
& \psi_{i}\left(x_{1}, \ldots, x_{i}\right):=e^{-t^{\prime} T(1+\varepsilon) \mu} \operatorname{Tr}\left(\exp \left(\sum_{j=1}^{i} t^{\prime} x_{j}\right)\right) \cdot\left\|\mathbf{E}_{X}\left(\exp \left(t^{\prime} X\right)\right)\right\|^{T-i}
\end{aligned}
$$

where $t^{\prime}=\log \left(\frac{(1+\varepsilon)(1-\mu)}{1-(1+\varepsilon) \mu}\right)$. If we choose $T>(2 \ln 2) \ln (2 n) /\left(\varepsilon^{2} \mu\right)=(2 \ln 2) n \ln (2 n) / \varepsilon^{2}$, then $\phi_{0}+$ $\psi_{0}<1$. Each $\phi_{i}, \psi_{i}$ can be computed efficiently and so one can find in polynomial time $\left(x_{1}, \ldots, x_{T}\right) \in$ $S_{\geq} \cap S_{\leq}$

\section{Comparing BSS and MMWUM}

In this section we show a striking similarity between the algorithms presented in Sections 4 and 6 The proof of Theorem 25]defines two potential functions for each iteration $t$.

$$
\begin{aligned}
& \Phi_{U}(t):=\operatorname{Tr} W_{U}(t)=\operatorname{Tr} \exp (\gamma A(t)) \\
& \Phi_{L}(t):=\operatorname{Tr} W_{L}(t)=\operatorname{Tr} \exp (-\gamma A(t))
\end{aligned}
$$

The proof shows that, for the algorithm of Section 6 , the potentials must change as follows:

$$
\begin{array}{ll}
\Phi_{U}(t+1) \leq\left(1+\delta_{U}\right) \Phi_{U}(t) & \forall t \in\{0, \ldots, T-1\} \\
\Phi_{L}(t+1) \leq\left(1-\delta_{L}\right) \Phi_{L}(t) & \forall t \in\{0, \ldots, T-1\} .
\end{array}
$$

Instead of requiring these potentials to grow and shrink in this way, we could instead parameterize the potential functions by the iteration number $t$ and then simply require that the potential do not grow from iteration to iteration. To formalize this alternative approach, let us define the new potential functions

$$
\begin{aligned}
& \Psi^{u}(A):=\operatorname{Tr} \exp (-u I+\gamma A) \\
& \Psi_{\ell}(A):=\operatorname{Tr} \exp (\ell I-\gamma A)
\end{aligned}
$$

and define the parameters $\Delta_{U}=\ln \left(1+\delta_{U}\right)$ and $\Delta_{L}=\ln \left(\left(1-\delta_{L}\right)^{-1}\right)$.

\footnotetext{
${ }^{1}$ There was an factor of $n$ in the $\phi_{i}$ that can be removed.
} 


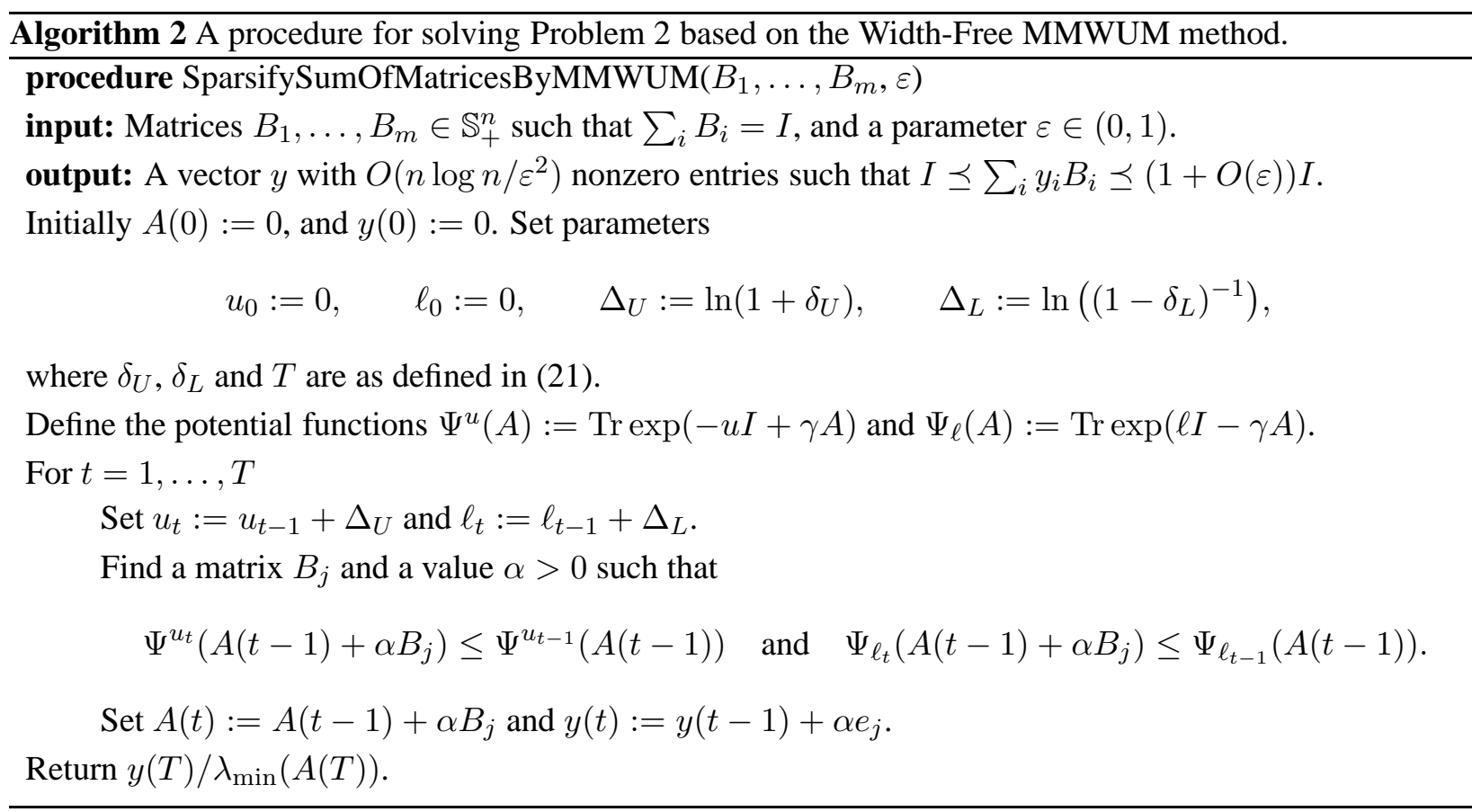

Proposition 28. The inequalities in (22) governing the algorithm's change in potentials are equivalent to inequalities in (23).

$$
\begin{aligned}
& \Psi^{(t+1) \Delta_{U}}\left(A(t)+\alpha B_{j}\right) \leq \Psi^{t \Delta_{U}}(A(t)) \\
& \Psi_{(t+1) \Delta_{L}}\left(A(t)+\alpha B_{j}\right) \leq \Psi_{t \Delta_{L}}(A(t))
\end{aligned}
$$

Proof. Obviously (22) is equivalent to

$$
\begin{array}{ll}
\left(1+\delta_{U}\right)^{-(t+1)} \cdot \Phi_{U}(t+1) \leq\left(1+\delta_{U}\right)^{-t} \cdot \Phi_{U}(t) & \forall t \in\{0, \ldots, T-1\}, \\
\left(1-\delta_{L}\right)^{-(t+1)} \cdot \Phi_{L}(t+1) \leq\left(1-\delta_{L}\right)^{-t} \cdot \Phi_{L}(t) & \forall t \in\{0, \ldots, T-1\} .
\end{array}
$$

By the definition of $\Phi_{U}$ and $\Phi_{L}$, and by properties of the exponential function, these inequalities are equivalent to

$$
\begin{aligned}
\operatorname{Tr} \exp \left(-(t+1) \Delta_{U} I+\gamma A(t+1)\right) & \leq \operatorname{Tr} \exp \left(-t \Delta_{U} I+\gamma A(t)\right), \\
\operatorname{Tr} \exp \left((t+1) \Delta_{L} I-\gamma A(t+1)\right) & \leq \operatorname{Tr} \exp \left(t \Delta_{L} I-\gamma A(t)\right) .
\end{aligned}
$$

Writing $A(t+1)=A(t)+\alpha B_{j}$, these inequalities in (24) are equivalent to (23).

Algorithm 2 gives pseudocode for the algorithm of Section 6, using the functions $\Psi^{u}$ and $\Psi_{\ell}$ to control the change in potentials.

The main point of this section is to observe that Algorithms 1 and 2 are identical with the exception of different parameters and different potential functions. We believe that this similarity between these two algorithms is intriguing, especially since the BSS algorithm has been called "highly original" by Naor [28]. In retrospect, it would have been perhaps more natural to develop the BSS algorithm by the following logical progression of ideas: first observe that MMWUM is useful for giving sparse solutions to SDPs, then design Algorithm 2, then later realize that a clever refinement of it leads to Algorithm 1 and its improved analysis. It is remarkable that Batson, Spielman and Srivastava developed their algorithm from first principles, apparently without knowing this connection to established algorithmic techniques. 
With the advantage of hindsight (i.e., the knowledge that the BSS algorithm exists), we now explain how one might be tempted to refine Algorithm 2 It is quite tempting to modify the potential functions to more strongly penalize eigenvalues which deviate from the desired range. The natural approach to do this would be to increase the derivatives of the potential function by increasing the parameter $\gamma$. However, as remarked at the end of Section 6 , the algorithm is actually unaffected by varying $\gamma$ ! Thus, to improve Algorithm 2 , one must seek a more substantially different potential function.

Focusing on the upper potential, we consider the question: is there a function $f: \mathbb{R} \rightarrow \mathbb{R}$ with steeper derivatives than $\exp (u-x)$ and such that, for any matrices $A$ and $B, \operatorname{Tr} f(A+B)$ can be easily related to $\operatorname{Tr} f(A)$ ? The natural candidates to try are $f(x)=-\log (u-x)$ and $f(x)=(u-x)^{-1}$ since, in both cases, $\operatorname{Tr} f(A+B)$ can be related to $\operatorname{Tr} f(A)$ by the Sherman-Morrison-Woodbury formula. We do not know whether the choice $f(x)=-\log (u-x)$ can be made to work. However, choosing $f(x)=(u-x)^{-1}$, one arrives at Algorithm 1, our generalization of the BSS algorithm. Of course, even after arriving at this algorithm, one must also analyze it, and this requires the delicate calculations that were accomplished by Batson, Spielman and Srivastava.

\section{Acknowledgements}

We thank Satyen Kale for helpful discussions.

\section{References}

[1] Rudolf Ahlswede and Andreas Winter. Strong converse for identification via quantum channels. IEEE Transactions on Information Theory, 48(3):569-579, March 2002.

[2] Ingo Althöfer. On sparse approximations to randomized strategies and convex combinations. Linear Algebra and Applications, 199:339-355, 1994.

[3] Sanjeev Arora and Satyen Kale. A combinatorial, primal-dual approach to semidefinite programs. In Proceedings of the 39th Annual ACM Symposium on Theory of Computing (STOC), 2007.

[4] Joshua Batson, Daniel A. Spielman, and Nikhil Srivastava. Twice-Ramanujan sparsifiers. In Proceedings of the 41st Annual ACM Symposium on Theory of Computing (STOC), 2009. To appear in SIAM Journal on Scientific Computing.

[5] András A. Benczúr and David R. Karger. Approximate $s$ - $t$ min-cuts in $\tilde{O}\left(n^{2}\right)$ time. In Proceedings of the 28th Annual ACM Symposium on Theory of Computing (STOC), 1996.

[6] András A. Benczúr and David R. Karger. Randomized approximation schemes for cuts and flows in capacitated graphs, 2002. http://arxiv.org/abs/cs/0207078.

[7] Moses Charikar, Chandra Chekuri, Ashish Goel, Sudipto Guha, and Serge A. Plotkin. Approximating a finite metric by a small number of tree metrics. In Proceedings of the 39th Annual IEEE Symposium on Foundations of Computer Science (FOCS), 1998.

[8] Marcel de Carli Silva and Levent Tunçel. Min-max theorems related to geometric representations of graphs and their SDPs, August 2011. http://arxiv.org/abs/1010.6036.

[9] Yoav Freund and Robert E. Schapire. Adaptive game playing using multiplicative weights. Games and Economic Behavior, 29:79-103, 1999. 
[10] Wai Shing Fung, Ramesh Hariharan, Nicholas J. A. Harvey, and Debmalya Panigrahi. A general framework for graph sparsification. In Proceedings of the 43rd Annual ACM Symposium on Theory of Computing (STOC), 2011.

[11] Wai Shing Fung and Nicholas J. A. Harvey. Graph sparsification by edge-connectivity and random spanning trees, May 2010. http://arxiv.org/abs/1005.0265

[12] Ashish Goel, Michael Kapralov, and Sanjeev Khanna. Graph sparsification via refinement sampling, April 2010. http://arxiv.org/abs/1004.4915.

[13] William W. Hager. Updating the inverse of a matrix. SIAM Review, 31(2):221-239, 1989.

[14] Ramesh Hariharan and Debmalya Panigrahi. A general framework for graph sparsification, April 2010. http://arxiv.org/abs/1004.4080

[15] Ramesh Hariharan and Debmalya Panigrahi. A linear-time algorithm for sparsification of unweighted graphs, May 2010. http://arxiv.org/abs/1005.0670

[16] Nicholas J. A. Harvey. Lecture notes for C\&O 750: Randomized algorithms, 2011. http://www.math.uwaterloo.ca/ 〜harvey/w11/Lecture11Notes.pdf.

[17] Thomas Hofmeister and Hanno Lefmann. Computing sparse approximations deterministically. Linear Algebra and its Applications, 240:9-19, 1996.

[18] Roger A. Horn and Charles R. Johnson. Matrix analysis. Cambridge University Press, Cambridge, 1990. Corrected reprint of the 1985 original.

[19] Garud Iyengar, David J. Phillips, and Clifford Stein. Approximation algorithms for semidefinite packing problems with applications to maxcut and graph coloring. In Michael Jünger and Volker Kaibel, editors, Integer Programming and Combinatorial Optimization, volume 3509 of Lecture Notes in Computer Science, pages 77-90. Springer Berlin / Heidelberg, 2005.

[20] Garud Iyengar, David J. Phillips, and Clifford Stein. Approximating semidefinite packing programs. SIAM Journal on Optimization, 21(1):231-268, 2011.

[21] Rahul Jain and Penghui Yao. A parallel approximation algorithm for positive semidefinite programming. In The 52nd Annual IEEE Symposium on Foundations of Computer Science (FOCS 2011), 2011. (to appear).

[22] Satyen Kale. Efficient Algorithms using the Multiplicative Weights Update Method. PhD thesis, Princeton University, 2007. Princeton Tech Report TR-804-07.

[23] Jonathan A. Kelner and Alex Levin. Spectral sparsification in the semi-streaming setting. In Proceedings of the 28th International Symposium on Theoretical Aspects of Computer Science (STACS), pages 440-451, 2011.

[24] Ioannis Koutis, Gary L. Miller, and Richard Peng. Approaching optimality for solving SDD systems. In Proceedings of the 51st Annual IEEE Symposium on Foundations of Computer Science (FOCS), 2010 .

[25] Richard J. Lipton and Neal E. Young. Simple strategies for large zero-sum games with applications to complexity theory. In Proceedings of the 26th Annual ACM Symposium on Theory of Computing (STOC), 1994. 
[26] Laszlo Lovász. Semidefinite programs and combinatorial optimization.

[27] R. J. McEliece, E. R. Rodemich, and H. C. Rumsey, Jr. The Lovász bound and some generalizations. J. Combin. Inform. System Sci., 3(3):134-152, 1978.

[28] Assaf Naor. Sparse quadratic forms and their geometric applications (after batson, spielman and srivastava). In Séminaire Bourbaki, 2011. Exposé no. 1033.

[29] Ilan Newman and Yuri Rabinovich. Finite volume spaces and sparsification, 2010. http://arxiv.org/abs/1002.3541.

[30] Lorenzo Orecchia and Nisheeth K. Vishnoi. Towards an SDP-based approach to spectral methods: A nearly-linear time algorithm for graph partitioning and decomposition. In Proceedings of the 22nd Annual ACM-SIAM Symposium on Discrete Algorithms (SODA), pages 532-545, 2011.

[31] Juan A. Rodríguez. On the Laplacian eigenvalues and metric parameters of hypergraphs. Linear Multilinear Algebra, 50(1):1-14, 2002.

[32] Mark Rudelson. Random vectors in the isotropic position. J. of Functional Analysis, 164(1):60-72, 1999.

[33] Mark Rudelson and Roman Vershynin. Sampling from large matrices: An approach through geometric functional analysis. Journal of the ACM, 54(4), 2007.

[34] Gideon Schechtman. Tight embedding of subspaces of $L_{p}$ in $\ell_{p}^{n}$ for even $p$. Proceedings of the AMS. To appear.

[35] Alexander Schrijver. A comparison of the Delsarte and Lovász bounds. IEEE Trans. Inform. Theory, 25(4):425-429, 1979.

[36] Anthony Man-Cho So, Yinyu Ye, and Jiawei Zhang. A unified theorem on SDP rank reduction. Mathematics of Operations Research, 33(4):910-920, 2008.

[37] Daniel A. Spielman and Nikhil Srivastava. An elementary proof of the restricted invertibility theorem. Israel J. Math. To appear.

[38] Daniel A. Spielman and Nikhil Srivastava. Graph sparsification by effective resistances. In Proceedings of the 40th Annual ACM Symposium on Theory of Computing (STOC), pages 563-568, 2008.

[39] Daniel A. Spielman and Shang-Hua Teng. Nearly-linear time algorithms for graph partitioning, graph sparsification, and solving linear systems. In Proceedings of the 36th Annual ACM Symposium on Theory of Computing (STOC), pages 81-90, 2004.

[40] Nikhil Srivastava. On contact points of convex bodies, 2009. http://www.cs.yale.edu/homes/srivastava/papers/contact.pdf.

[41] Roman Vershynin. A note on sums of independent random matrices after Ahlswede-Winter, 2008. http://www-personal.umich.edu/ romanv/teaching/reading-group/ahlswede-winter.pdf

[42] Avi Wigderson and David Xiao. Derandomizing the Ahlswede-Winter matrix-valued Chernoff bound using pessimistic estimators and applications. Theory of Computing, 4(3), 2008.

[43] Neal Young. Greedy algorithms by derandomizing unknown distributions. Technical Report 1087, Department of ORIE, Cornell University, March 1994. http://hdl . handle. net/1813/8971. 
[44] Neal Young. Randomized rounding without solving the linear program. In Proceedings of the 6th Annual ACM-SIAM Symposium on Discrete Algorithms (SODA), pages 170-178, 1995. 


\section{A Proofs of the Applications}

Corollary 4. Let $G=(V, E)$ be a graph, let $w: E \rightarrow \mathbb{R}_{+}$be a weight function, and let $c_{1}, \ldots, c_{k}: E \rightarrow$ $\mathbb{R}_{+}$be cost functions, with $k=O(n)$. Let $\mathcal{L}_{G}(w)$ denote the Laplacian matrix for graph $G$ with weight function $w$. For any real $\varepsilon \in(0,1)$, there is a deterministic polynomial-time algorithm to find a subgraph $H$ of $G$ and a weight function $w_{H}: E(H) \rightarrow \mathbb{R}_{+}$such that

$$
\begin{aligned}
& \mathcal{L}_{G}(w) \preceq \mathcal{L}_{H}\left(w_{H}\right) \preceq(1+\varepsilon) \mathcal{L}_{G}(w), \\
& \sum_{e \in E} w_{e} c_{i, e} \leq \sum_{e \in E(H)} w_{H, e} c_{i, e} \leq(1+\varepsilon) \sum_{e \in E} w_{e} c_{i, e} \quad \text { for all } i
\end{aligned}
$$

and $|E(H)|=O\left(n / \varepsilon^{2}\right)$.

Proof. For every edge $e=i j \in E$, let $B_{e}$ be the direct sum $w_{i j}\left[\left(e_{i}-e_{j}\right)\left(e_{i}-e_{j}\right)^{T} \oplus c_{1, e} \oplus \cdots \oplus c_{k, e}\right]$. Let $B:=\mathcal{L}_{G}(w) \oplus w^{T} c_{1} \oplus \cdots \oplus w^{T} c_{k}$. The result follows immediately by applying Theorem 3 to these matrices.

Corollary 5. Let $G=(V, E)$ be a graph and let $w: E \rightarrow \mathbb{R}_{+}$be a weight function. Let $E_{1}, \ldots, E_{k}$ be a partition of the edges, i.e., each edge is colored with one of $k$ colors. For any real $\varepsilon \in(0,1)$, there is a deterministic polynomial-time algorithm to find a subgraph $H$ of $G$ and a weight function $w_{H}: E(H) \rightarrow$ $\mathbb{R}_{+}$such that

$$
\begin{aligned}
\mathcal{L}_{G}(w) \preceq \mathcal{L}_{H}\left(w_{H}\right) & \preceq(1+\varepsilon) \mathcal{L}_{G}(w), \\
(1-\varepsilon) \sum_{e \in E_{i}} w_{e} \leq \sum_{e \in E(H) \cap E_{i}} w_{H, e} & \leq(1+\varepsilon) \sum_{e \in E_{i}} w_{e} \quad \text { for all } i,
\end{aligned}
$$

and $|E(H)|=O\left((n+k) / \varepsilon^{2}\right)$.

Proof. For each $i$, let $c_{i}: E \rightarrow \mathbb{R}$ be the characteristic vector of $E_{i}$. Now apply Corollary 4 ,

Corollary 6 (Spectral sparsifiers for hypergraphs). For any real $\varepsilon \in(0,1)$, there is a deterministic polynomial-time algorithm to find a sub-hypergraph $\mathcal{G}$ of $\mathcal{H}$ and a weight function $w_{\mathcal{G}}: \mathcal{E}(\mathcal{G}) \rightarrow \mathbb{R}_{+}$such that

$$
\mathcal{L}_{\mathcal{H}}(w) \preceq \mathcal{L}_{\mathcal{G}}\left(w_{\mathcal{G}}\right) \preceq(1+\varepsilon) \mathcal{L}_{\mathcal{H}}(w),
$$

and $|\mathcal{E}(\mathcal{G})|=O\left(n / \varepsilon^{2}\right)$.

Proof. The result follows directly by applying Theorem 3 to the matrices $w_{E} \mathcal{L}_{E}$.

Corollary 7 (Cut sparsifiers for hypergraphs, second definition). For any real $\varepsilon \in(0,1)$, there is a deterministic polynomial-time algorithm to find a sub-hypergraph $\mathcal{G}$ of $\mathcal{H}$ and a weight function $w_{\mathcal{G}}: \mathcal{E}(\mathcal{G}) \rightarrow \mathbb{R}_{+}$ such that

$$
w^{*}\left(\delta_{\mathcal{H}}(S)\right) \leq w_{\mathcal{G}}^{*}\left(\delta_{\mathcal{G}}(S)\right) \leq(1+\varepsilon) w^{*}\left(\delta_{\mathcal{H}}(S)\right) \quad \text { for every } S \subseteq V,
$$

and $|\mathcal{E}(\mathcal{G})|=O\left(n / \varepsilon^{2}\right)$.

Proof. Note that $w^{*}\left(\delta_{\mathcal{H}}(S)\right)$ is obtained by evaluating the quadratic form $x^{T} \mathcal{L}_{\mathcal{H}}(w) x$, where $x$ is the characteristic vector of $S$. Thus the sparsifier produced by Corollary 6 satisfies the desired inequalities. 
Corollary 8 (Cut sparsifiers for hypergraphs, first definition). Assume that $\mathcal{H}$ is an r-uniform hypergraph. For any real $\varepsilon \in(0,1)$, there is a deterministic polynomial-time algorithm to find a sub-hypergraph $\mathcal{G}$ of $\mathcal{H}$ and a weight function $w_{\mathcal{G}}: \mathcal{E}(\mathcal{G}) \rightarrow \mathbb{R}_{+}$such that

$$
\frac{(r-1)}{r^{2} / 4} w\left(\delta_{\mathcal{H}}(S)\right) \leq w_{\mathcal{G}}\left(\delta_{\mathcal{G}}(S)\right) \leq \frac{(1+\varepsilon) r^{2}}{4(r-1)} w\left(\delta_{\mathcal{H}}(S)\right) \quad \forall S \subseteq V
$$

and $|\mathcal{E}(\mathcal{G})|=O\left(n / \varepsilon^{2}\right)$. In other words, the sparsified hypergraph $\mathcal{G}$ approximates the weight of the cuts in the hypergraph $\mathcal{H}$ to within a factor $\Theta\left(r^{2}\right)$.

Proof. For any $r$-uniform hypergraph $\mathcal{H}$, it is easy to see that

$$
(r-1) w\left(\delta_{\mathcal{H}}(S)\right) \leq w^{*}\left(\delta_{\mathcal{H}}(S)\right) \leq\lfloor r / 2\rfloor\lceil r / 2\rceil w\left(\delta_{\mathcal{H}}(S)\right) \quad \forall S \subseteq V .
$$

Thus the sparsifier produced by Corollary 6 satisfies the desired inequalities.

Corollary 9 (Cut sparsifiers for 3-uniform hypergraphs). Assume that $\mathcal{H}$ is a 3-uniform hypergraph. For any $\varepsilon \in(0,1)$, there is a deterministic polynomial-time algorithm to find a sub-hypergraph $\mathcal{G}$ of $\mathcal{H}$ and $a$ weight function $w_{\mathcal{G}}: \mathcal{E}(\mathcal{G}) \rightarrow \mathbb{R}_{+}$such that

$$
w\left(\delta_{\mathcal{H}}(S)\right) \leq w_{\mathcal{G}}\left(\delta_{\mathcal{G}}(S)\right) \leq(1+\varepsilon) w\left(\delta_{\mathcal{H}}(S)\right) \quad \forall S \subseteq V
$$

and $|\mathcal{E}(\mathcal{G})|=O\left(n / \varepsilon^{2}\right)$.

Proof. Since $r=3$, a consequence of (25) is that $w^{*}\left(\delta_{\mathcal{H}}(S)\right)=2 w\left(\delta_{\mathcal{H}}(S)\right)$ for every $S$. Thus the sparsifier produced by Corollary 6 satisfies the desired inequalities.

Corollary 10. Let $A_{1}, \ldots, A_{m}$ be symmetric, positive semidefinite matrices of size $n \times n$, and let $B$ be a symmetric matrix of size $n \times n$. Let $c \in \mathbb{R}^{m}$ with $c \geq 0$. Suppose that the semidefinite program (SDP)

$$
\min \left\{c^{T} z: \sum_{i} z_{i} A_{i} \succeq B, z \in \mathbb{R}^{m}, z \geq 0\right\}
$$

has a feasible solution $z^{*}$. Then, for any real $\varepsilon \in(0,1)$, it has a feasible solution $\bar{z}$ with at most $O\left(n / \varepsilon^{2}\right)$ nonzero entries and $c^{T} \bar{z} \leq(1+\varepsilon) c^{T} z^{*}$.

Proof. Let $B_{i}^{\prime}:=\left[\begin{array}{cc}z_{i}^{*} A_{i} & \mathbf{0} \\ \mathbf{0} & c_{i} z_{i}^{*}\end{array}\right]$ for every $i \in[m]$ and $B^{\prime}:=\left[\begin{array}{cc}D & \mathbf{0} \\ \mathbf{0} & c^{T} z^{*}\end{array}\right]$, where $D:=\sum_{i} z_{i}^{*} A_{i} \succeq B$. Then $B_{i}^{\prime} \succeq 0$ and $B^{\prime}=\sum_{i} B_{i}^{\prime}$. By applying Theorem 3, we obtain $y \in \mathbb{R}^{m}$ with $y \geq 0$ and $O\left(n / \varepsilon^{2}\right)$ nonzero entries such that $\sum_{i} y_{i} z_{i}^{*} A_{i} \succeq D \succeq B$ and $\sum_{i} c_{i} y_{i} z_{i}^{*} \leq(1+\varepsilon) c^{T} z^{*}$. Thus, we can take $\bar{z}_{i}=y_{i} z_{i}^{*}$ for every $i \in[m]$.

Corollary 11. Let $G=(V, E)$ be a graph. For any real $\varepsilon \in(0,1)$, there is a deterministic polynomial-time algorithm to find a subgraph $H$ of $G$ such that

$$
(1-\varepsilon) t^{\prime}(G) \leq t^{\prime}(H) \leq t^{\prime}(G)
$$

and $|E(H)|=O\left(n / \varepsilon^{2}\right)$. 
Proof. It is straightforward to formulate $t^{\prime}(G)$ as an SDP (see, e.g., [26]) so that its dual has an optimal solution and there is no duality gap. The dual can be written as:

$$
\max \left\{\sum_{e \in E} z_{e}: \operatorname{Diag}(y) \succeq \mathcal{L}_{G}(z), \sum_{v \in V} y_{v}=1, z \geq 0\right\}
$$

The proof is now almost identical to the proof of Corollary 10 Let $\left(z^{*}, y^{*}\right)$ be an optimal solution. Using Theorem 3, we obtain $\bar{z} \in \mathbb{R}^{E}$ with $\bar{z} \geq 0$ and $O\left(n / \varepsilon^{2}\right)$ nonzero entries such that $\left(y^{*}, \bar{z}\right)$ is feasible in (26) and has objective value $\sum_{e \in E(H)} \bar{z}_{e} \geq(1-\varepsilon) t(G)$, where $H=(V, E(H))$ and $E(H)$ is the support of $\bar{z}$. Then $\bar{z}$ is also feasible for the SDP defined using $H$ instead of $G$, which shows that $t^{\prime}(H) \geq$ $(1-\varepsilon) t^{\prime}(G)$.

Corollary 12, Let $G=(V, E)$ be a graph. For any real $\varepsilon \in(0,1)$, there is a deterministic polynomial-time algorithm to find a supergraph $H$ of $G$ such that

$$
\frac{\vartheta^{\prime}(G)}{1-\varepsilon+\varepsilon \vartheta^{\prime}(G)} \leq \vartheta^{\prime}(H) \leq \vartheta^{\prime}(G)
$$

and $|E(H)|=\left(\begin{array}{l}n \\ 2\end{array}\right)-O\left(n / \varepsilon^{2}\right)$.

Proof. For a graph $G=(V, E)$, define $t(G)$ as the square of the minimum radius of a hypersphere on $\mathbb{R}^{n}$ such that there is a map from $V$ to the hypersphere such that adjacent vertices are mapped to points at distance exactly 1 . Lovász [26] noted that $t(G)$ is related to the Lovász theta number $\vartheta(\bar{G})$ of the complement $\bar{G}$ of $G$ by the formula $2 t(G)+1 / \vartheta(\bar{G})=1$; see [8] for a proof. By repeating the same proof for $t^{\prime}(G)$, one finds that $2 t^{\prime}(G)+1 / \vartheta^{\prime}(\bar{G})=1$. The result now follows from Corollary 11 via this formula.

Corollary 13, Let $G$ be a graph such that $\vartheta^{\prime}(G)=o(\sqrt{n})$. For any real $\gamma>0$, there is a supergraph $H$ of G such that

$$
\frac{\vartheta^{\prime}(G)}{1+\gamma} \leq \vartheta^{\prime}(H) \leq \vartheta^{\prime}(G)
$$

and $|E(H)|=\left(\begin{array}{l}n \\ 2\end{array}\right)-O\left(n \vartheta(G)^{2} / \gamma^{2}\right)$.

Proof. Apply Corollary 12 with $\varepsilon:=\gamma / \vartheta^{\prime}(G)$.

Corollary 14, Let $G$ be a graph such that $\vartheta^{\prime}(G)=\Omega(\sqrt{n})$. For any real $\gamma \geq 1$, there is a supergraph $H$ of $G$ such that

$$
\vartheta^{\prime}(H)=\Omega(\sqrt{n} / \gamma)
$$

and $|E(H)|=\left(\begin{array}{l}n \\ 2\end{array}\right)-O\left(n^{2} / \gamma^{2}\right)$.

Proof. Apply Corollary 12 with $\varepsilon:=\gamma / \sqrt{n}$.

Corollary 16. Let $B_{1}, \ldots, B_{m}$ be symmetric, positive semidefinite matrices of size $n \times n$ and let $\lambda \in \mathbb{R}^{m}$ satisfy $\lambda \geq 0$ and $\sum_{i} \lambda_{i}=1$. Let $B=\sum_{i} \lambda_{i} B_{i}$. For any $\varepsilon \in(0,1)$, there exists $\mu \geq 0$ with $\sum_{i} \mu_{i}=1$ such that $\mu$ has $O\left(n / \varepsilon^{2}\right)$ nonzero entries and

$$
(1-\varepsilon) B \preceq \sum_{i} \mu_{i} B_{i} \preceq(1+\varepsilon) B .
$$


Proof. Let $B_{i}^{\prime}:=\left[\begin{array}{cc}\lambda_{i} B_{i} & \mathbf{0} \\ \mathbf{0} & \lambda_{i}\end{array}\right]$ for every $i \in[m]$ and $B^{\prime}:=\left[\begin{array}{cc}B & \mathbf{0} \\ \mathbf{0} & 1\end{array}\right]$, so that $B_{i}^{\prime} \succeq 0$ and $B^{\prime}=\sum_{i} B_{i}^{\prime}$. By applying Theorem 3 , we obtain $y \in \mathbb{R}^{m}$ with $y \geq 0$ and $O\left(n / \varepsilon^{2}\right)$ nonzero entries such that $B^{\prime} \preceq \sum_{i} y_{i} B_{i}^{\prime} \preceq$ $(1+\varepsilon) B^{\prime}$ or, equivalently, $B \preceq \sum_{y} y_{i} \lambda_{i} B_{i} \preceq(1+\varepsilon) B$ and $1 \leq \sum_{i} y_{i} \lambda_{i} \leq 1+\varepsilon$. Let $\mu \in \mathbb{R}^{m}$ be defined by $\mu_{i}:=y_{i} \lambda_{i} /\left(\sum_{i} y_{i} \lambda_{i}\right)$. Then $\mu \geq 0$ and $\sum_{i} \mu_{i}=1$, and

$$
(1-\varepsilon) B \preceq \frac{B}{1+\varepsilon} \preceq \frac{B}{\sum_{i} y_{i} \lambda_{i}} \preceq \sum_{i} \mu_{i} B_{i} \preceq \frac{1+\varepsilon}{\sum_{i} y_{i} \lambda_{i}} B \preceq(1+\varepsilon) B .
$$

This completes the proof.

Corollary 17, Let $G=(V, E)$ be a graph, let $w: E \rightarrow \mathbb{R}_{+}$be a weight function, and let $\mathcal{F}$ be a collection of subgraphs of $G$ such that $\sum_{F \in \mathcal{F}}|V(F)|=O(n)$. For any real $\varepsilon \in(0,1)$, there is a deterministic polynomial-time algorithm to find a subgraph $H$ of $G$ and a weight function $w_{H}: E(H) \rightarrow \mathbb{R}_{+}$such that $|E(H)|=O\left(n / \varepsilon^{2}\right)$ and

$$
\begin{gathered}
\mathcal{L}_{G}(w) \preceq \mathcal{L}_{H}\left(w_{H}\right) \preceq(1+\varepsilon) \mathcal{L}_{G}(w), \\
\mathcal{L}_{F}\left(w_{F}\right) \preceq \mathcal{L}_{H \cap F}\left(w_{H} \uparrow_{E(H \cap F)}\right) \preceq(1+\varepsilon) \mathcal{L}_{F}\left(w_{F}\right) \quad \text { for all } F \in \mathcal{F},
\end{gathered}
$$

where $w_{F}:=w \uparrow_{E(F)}$ is the restriction of $w$ to the coordinates $E(F)$ and $H \cap F=(V(F), E(F) \cap E(H))$.

Proof. For each edge $e \in E$, define $B_{e}:=w_{e}\left[\mathcal{L}_{G}\left(\chi^{e}\right) \oplus \bigoplus_{F \in \mathcal{F}} \mathcal{L}_{F}\left(\chi^{e} \uparrow_{E(F)}\right)\right]$, where $\chi^{e}$ denotes the characteristic vector of $\{e\}$ as a subset of $E$. Now apply Theorem 3 .

\section{B The MMWUM}

In this section we provide some proofs about the MMWUM. These proofs are due to Kale [22]. Our set up and conclusions are slightly different and we modified the proofs accordingly. We reproduce the proofs here for the sake of completeness.

Theorem 22] can be viewed as a block-friendly version of MMWUM. First we show the version with only one block. It is basically the same as [22, Theorem 13 in Chapter 4].

Theorem 29. Let $T$ be a positive integer. Let $C, A_{1}, \ldots, A_{m} \in \mathbb{S}^{n}$. Let $\eta>0$ and $0<\beta \leq 1 / 2$. For any given $X \in \mathbb{S}^{n}$, consider the system

$$
\sum_{i=1}^{m} y_{i}\left\langle A_{i}, X\right\rangle \geq\langle C, X\rangle-\eta \operatorname{Tr} X, \quad \text { and } \quad y \in \mathbb{R}_{+}^{m} .
$$

Let $\{\mathcal{P}, \mathcal{N}\}$ be a partition of $[T]$, let $0<\ell \leq \rho$, and let $W^{(t)} \in \mathbb{S}^{n}$ and $\ell^{(t)} \in \mathbb{R}$ for $t \in[T+1]$. Let $y^{(t)} \in \mathbb{R}^{m}$ for $t \in[T]$. Suppose the following properties hold:

$$
\begin{aligned}
& W^{(t+1)}= \exp \left(-\frac{\beta}{\ell+\rho} \sum_{\tau=1}^{t}\left[\sum_{i=1}^{m} y_{i}^{(\tau)} A_{i}-C+\ell^{(\tau)} I\right]\right), \quad \forall t \in\{0, \ldots, T\}, \\
& y=y^{(t)} \text { is a solution for }\left[27 \text { with } X=W^{(t)},\right. \forall t \in[T], \\
& \sum_{i=1}^{m} y_{i}^{(t)} A_{i}-C \in\left\{\begin{array}{ll}
{[-\ell, \rho],} & \text { if } t \in \mathcal{P}, \\
{[-\rho, \ell],} & \text { if } t \in \mathcal{N},
\end{array} \quad \forall t \in[T],\right. \\
& \ell^{(t)}=\ell, \quad \forall t \in \mathcal{P}, \quad \text { and } \quad \ell^{(t)}=-\ell, \quad \forall t \in \mathcal{N} .
\end{aligned}
$$


Define $\bar{y}:=\frac{1}{T} \sum_{t=1}^{T} y^{(t)}$. Then

$$
\sum_{i=1}^{m} \bar{y}_{i} A_{i}-C \succeq-\left[\beta \ell+\frac{(\rho+\ell) \ln n}{T \beta}+(1+\beta) \eta\right] I .
$$

The main tool for the proof of Theorem 29 is the following result:

Theorem 30 (Kale [22, Corollary 3 in Chapter 3]). Let $0<\beta \leq 1 / 2$. Let $T$ be a positive integer. Let $\{\mathcal{P}, \mathcal{N}\}$ be a partition of $[T]$, and let $M^{(t)} \in \mathbb{S}^{n}$ for $t \in[T]$ and $W^{(t)} \in \mathbb{S}^{n}$ for $t \in[T+1]$ with the following properties:

$$
\begin{gathered}
W^{(t+1)}=\exp \left(-\beta \sum_{\tau=1}^{t} M^{(\tau)}\right) \quad \forall t=0, \ldots, T, \\
0 \preceq M^{(t)} \preceq I, \quad \forall t \in \mathcal{P}, \quad \text { and } \quad-I \preceq M^{(t)} \preceq 0, \quad \forall t \in \mathcal{N},
\end{gathered}
$$

Let

$$
P^{(t)}:=\frac{1}{\operatorname{Tr} W^{(t)}} W^{(t)}, \quad \forall t \in[T]
$$

Then

$$
(1-\beta) \sum_{t \in \mathcal{P}}\left\langle M^{(t)}, P^{(t)}\right\rangle+(1+\beta) \sum_{t \in \mathcal{N}}\left\langle M^{(t)}, P^{(t)}\right\rangle \leq \lambda_{\min }\left(\sum_{t=1}^{T} M^{(t)}\right)+\frac{\ln n}{\beta} .
$$

Proof. Set $\Phi^{(t)}:=\operatorname{Tr}\left(W^{(t)}\right)$ for $t \in[T+1]$. Put $\beta_{1}:=1-e^{-\beta}$ and $\beta_{2}:=e^{\beta}-1$. Then, for any $t \in[T]$,

$$
\begin{aligned}
\Phi^{(t+1)} & =\operatorname{Tr}\left(W^{(t+1)}\right)=\operatorname{Tr}\left(\exp \left(-\beta \sum_{\tau=1}^{t} M^{(\tau)}\right)\right) \\
& \leq \operatorname{Tr}\left(\exp \left(-\beta \sum_{\tau=1}^{t-1} M^{(\tau)}\right) \exp \left(-\beta M^{(t)}\right)\right)=\operatorname{Tr}\left(W^{(t)} \exp \left(-\beta M^{(t)}\right)\right) \\
& =\left\langle W^{(t)}, \exp \left(-\beta M^{(t)}\right)\right\rangle,
\end{aligned}
$$

where we have used Golden-Thompson's inequality (14).

Using the fact that $e^{x}$ is convex, one can prove that

$$
\begin{aligned}
& 0 \preceq A \preceq I \Longrightarrow \exp (-\beta A) \preceq I-\beta_{1} A, \\
&-I \preceq A \preceq 0 \Longrightarrow \exp (-\beta A) \preceq I-\beta_{2} A .
\end{aligned}
$$

Suppose that $t \in \mathcal{P}$. Then $\exp \left(-\beta M^{(t)}\right) \preceq I-\beta_{1} M^{(t)}$, and since $W^{(t)} \succeq 0$, we get

$$
\begin{aligned}
\Phi^{(t+1)} & \leq\left\langle W^{(t)}, \exp \left(-\beta M^{(t)}\right)\right\rangle \leq\left\langle W^{(t)}, I-\beta_{1} M^{(t)}\right\rangle \\
& =\operatorname{Tr}\left(W^{(t)}\right)-\beta_{1}\left\langle W^{(t)}, M^{(t)}\right\rangle \\
& =\operatorname{Tr}\left(W^{(t)}\right)-\operatorname{Tr}\left(W^{(t)}\right) \beta_{1}\left\langle P^{(t)}, M^{(t)}\right\rangle \\
& =\operatorname{Tr}\left(W^{(t)}\right)\left[1-\beta_{1}\left\langle P^{(t)}, M^{(t)}\right\rangle\right] \\
& =\Phi^{(t)}\left[1-\beta_{1}\left\langle P^{(t)}, M^{(t)}\right\rangle\right] \\
& \leq \Phi^{(t)} \exp \left(-\beta_{1}\left\langle P^{(t)}, M^{(t)}\right\rangle\right) .
\end{aligned}
$$


Similarly, if $t \in \mathcal{N}$, then

$$
\Phi^{(t+1)} \leq \Phi^{(t)} \exp \left(-\beta_{2}\left\langle P^{(t)}, M^{(t)}\right\rangle\right)
$$

By induction on $t$, and using $\Phi^{(1)}=\operatorname{Tr}(I)=n$, we get

$$
\Phi^{(t+1)} \leq n \exp \left(-\beta_{1} \sum_{\tau \in \mathcal{P} \cap[t]}\left\langle M^{(\tau)}, P^{(\tau)}\right\rangle-\beta_{2} \sum_{\tau \in \mathcal{N} \cap[t]}\left\langle M^{(\tau)}, P^{(\tau)}\right\rangle\right), \quad \forall t \in[T] .
$$

For every $A \in \mathbb{S}^{n}$, we have $\operatorname{Tr}(\exp (A))=\sum_{i=1}^{n} e^{\lambda_{i}} \geq e^{\lambda_{j}}$ for any $j \in[n]$, where $\lambda_{1}, \ldots, \lambda_{n}$ are the eigenvalues of $A$. Thus,

$$
\begin{aligned}
\Phi^{(T+1)} & =\operatorname{Tr}\left(W^{(T+1)}\right)=\operatorname{Tr}\left(\exp \left(-\beta \sum_{t=1}^{T} M^{(t)}\right)\right) \\
& \geq \exp \left(\lambda_{\max }\left(-\beta \sum_{t=1}^{T} M^{(t)}\right)\right)=\exp \left(-\beta \lambda_{\min }\left(\sum_{t=1}^{T} M^{(t)}\right)\right) .
\end{aligned}
$$

Thus,

$$
\exp \left[-\beta \lambda_{\min }\left(\sum_{t=1}^{T} M^{(t)}\right)\right] \leq n \exp \left[-\beta_{1} \sum_{t \in \mathcal{P}}\left\langle M^{(t)}, P^{(t)}\right\rangle-\beta_{2} \sum_{t \in \mathcal{N}}\left\langle M^{(t)}, P^{(t)}\right\rangle\right] .
$$

By taking $\ln (\cdot)$ on both sides, we get

$$
-\beta \lambda_{\min }\left(\sum_{t=1}^{T} M^{(t)}\right) \leq \ln n-\left[\beta_{1} \sum_{t \in \mathcal{P}}\left\langle M^{(t)}, P^{(t)}\right\rangle+\beta_{2} \sum_{t \in \mathcal{N}}\left\langle M^{(t)}, P^{(t)}\right\rangle\right],
$$

so

$$
\beta_{1} \sum_{t \in \mathcal{P}}\left\langle M^{(t)}, P^{(t)}\right\rangle+\beta_{2} \sum_{t \in \mathcal{N}}\left\langle M^{(t)}, P^{(t)}\right\rangle \leq \beta \lambda_{\min }\left(\sum_{t=1}^{T} M^{(t)}\right)+\ln n,
$$

and

$$
\frac{\beta_{1}}{\beta} \sum_{t \in \mathcal{P}}\left\langle M^{(t)}, P^{(t)}\right\rangle+\frac{\beta_{2}}{\beta} \sum_{t \in \mathcal{N}}\left\langle M^{(t)}, P^{(t)}\right\rangle \leq \lambda_{\min }\left(\sum_{t=1}^{T} M^{(t)}\right)+\frac{\ln n}{\beta} .
$$

Since $\sum_{t \in \mathcal{P}}\left\langle M^{(t)}, P^{(t)}\right\rangle \geq 0$ and $\sum_{t \in \mathcal{N}}\left\langle M^{(t)}, P^{(t)}\right\rangle \leq 0$, to prove 29] it suffices to show that $1-\beta \leq$ $\beta_{1} / \beta$ and $1+\beta \geq \beta_{2} / \beta$. It is not hard to prove that

$$
1-e^{-x} \geq x(1-x), \forall x \in[0,+\infty) \quad \text { and } \quad e^{x}-1 \leq x(1+x), \forall x \in\left[0, \frac{1}{2}\right]
$$

So our choice of $\beta_{1}$ and $\beta_{2}$ ensures that $1-\beta \leq \beta_{1} / \beta$ and $1+\beta \geq \beta_{2} / \beta$.

We can now show the proof of Theorem 29

Proof of Theorem 29 Let $M^{(t)}:=\frac{1}{\ell+\rho}\left[\sum_{i=1}^{m} y_{i}^{(t)} A_{i}-C+\ell^{(t)} I\right]$ and $P^{(t)}:=W^{(t)} / \operatorname{Tr} W^{(t)}$ for every $t$. For every $t \leq T$, using (27),

$$
\begin{aligned}
\left\langle M^{(t)}, P^{(t)}\right\rangle & =\frac{1}{\ell+\rho}\left[\sum_{i=1}^{m} y_{i}^{(t)}\left\langle A_{i}, P^{(t)}\right\rangle-\left\langle C, P^{(t)}\right\rangle+\ell^{(t)}\left\langle I, P^{(t)}\right\rangle\right] \\
& =\frac{1}{(\ell+\rho) \operatorname{Tr} W^{(t)}}\left[\sum_{i=1}^{m} y_{i}^{(t)}\left\langle A_{i}, W^{(t)}\right\rangle-\left\langle C, W^{(t)}\right\rangle\right]+\frac{\ell^{(t)}}{\ell+\rho} \geq-\frac{\eta}{\ell+\rho}+\frac{\ell^{(t)}}{\ell+\rho}
\end{aligned}
$$


since $y^{(t)}$ is a solution for (27) with $X:=W^{(t)}$. Thus, by (29),

$$
\begin{aligned}
& \sum_{t \in \mathcal{P}} \frac{(1-\beta)\left(\ell^{(t)}-\eta\right)}{\ell+\rho}+\sum_{t \in \mathcal{N}} \frac{(1+\beta)\left(\ell^{(t)}-\eta\right)}{\ell+\rho} \\
& \quad \leq \frac{1}{\rho+\ell} \lambda_{\min }\left(\sum_{t=1}^{T}\left[\left(\sum_{i=1}^{m} y_{i}^{(t)} A_{i}\right)-C+\ell^{(t)} I\right]\right)+\frac{\ln n}{\beta} .
\end{aligned}
$$

Multiply through by $\ell+\rho$ and move $\ell^{(t)} I$ out of $\lambda_{\min }(\cdot)$ :

$$
\begin{aligned}
\sum_{t \in \mathcal{P}}(1-\beta) \ell^{(t)}+\sum_{t \in \mathcal{N}}(1+\beta) \ell^{(t)} & -T(1+\beta) \eta \\
& \leq \lambda_{\min }\left(\sum_{t=1}^{T}\left[\left(\sum_{i=1}^{m} y_{i}^{(t)} A_{i}\right)-C\right]\right)+\left(\sum_{t=1}^{T} \ell^{(t)}\right)+\frac{(\rho+\ell) \ln n}{\beta} .
\end{aligned}
$$

Thus,

$$
\sum_{t \in \mathcal{P}}-\beta \ell^{(t)}+\sum_{t \in \mathcal{N}} \beta \ell^{(t)} \leq \lambda_{\min }\left(\sum_{t=1}^{T}\left[\left(\sum_{i=1}^{m} y_{i}^{(t)} A_{i}\right)-C\right]\right)+\frac{(\rho+\ell) \ln n}{\beta}+T(1+\beta) \eta .
$$

Next note that $\sum_{t \in \mathcal{P}}-\ell^{(t)}+\sum_{t \in \mathcal{N}} \ell^{(t)}=\sum_{t \in \mathcal{P}}-\ell+\sum_{t \in \mathcal{N}}-\ell=-T \ell$, so

$$
0 \leq \lambda_{\min }\left(\sum_{t=1}^{T}\left[\left(\sum_{i=1}^{m} y_{i}^{(t)} A_{i}\right)-C\right]\right)+\beta T \ell+\frac{(\rho+\ell) \ln n}{\beta}+T(1+\beta) \eta .
$$

and

$$
0 \leq \lambda_{\min }\left(\frac{1}{T} \sum_{t=1}^{T}\left[\left(\sum_{i=1}^{m} y_{i}^{(t)} A_{i}\right)-C\right]\right)+\beta \ell+\frac{(\rho+\ell) \ln n}{T \beta}+(1+\beta) \eta
$$

Thus,

$$
\sum_{i=1}^{m} \bar{y}_{i} A_{i}-C=\frac{1}{T} \sum_{t=1}^{T}\left[\left(\sum_{i=1}^{m} y_{i}^{(t)} A_{i}\right)-C\right] \succeq-\left[\beta \ell+\frac{(\rho+\ell) \ln n}{T \beta}+(1+\beta) \eta\right] I .
$$

Theorem 22 can be easily proved from Theorem 29 . First, we apply Theorem 29 separately for each block. In each iteration, $y^{(t)}$ is a solution for (27) for all blocks simultaneously, and so the conclusion in (28) holds for all blocks with same $\bar{y}$. This new algorithm can be seen as equivalent to running $K$ copies of MMWUM, each with different input data, with the caveat that all copies run for the same number of iterations and the vector $y^{(t)}$ returned from the oracle is the same for all copies at each iteration $t$.

\section{Optimality of MMWUM Oracle}

Proposition 24. Any oracle for satisfying (9) must have $\rho=\Omega(n / \eta)$, even if the $B_{i}$ matrices have rank one, and even if $X_{1}$ is a scalar multiple of $X_{2}^{-1}$. 
Proof. Let $k=n / 3$, let $I_{k}$ be the identity of size $k \times k$, and let $e_{j} \in \mathbb{R}^{k}$ be the $j$ th standard basis vector. Let $\zeta=3 \eta$ and define

$$
X_{1}=\operatorname{Diag}\left(1, \zeta^{3}, \zeta\right) \otimes I_{k}, \quad X_{2}=\operatorname{Diag}\left(1,1 / \zeta^{3}, 1 / \zeta\right) \otimes I_{k},
$$

where $\otimes$ denotes tensor product. For $j=1, \ldots, k$, define

$$
v_{1, j}=[1 / \sqrt{2},-1 / \sqrt{2}, 0] \otimes e_{j}, \quad v_{2, j}=[1 / \sqrt{2}, 1 / \sqrt{2}, 0] \otimes e_{j}, \quad v_{3, j}=[0,0,1] \otimes e_{j} .
$$

Let $B_{i, j}=v_{i, j} v_{i, j}^{T}$. Note that $\sum_{i, j} B_{i, j}=I$.

The oracle cannot choose a matrix $B_{i, j}$ with $i \in\{1,2\}$, since satisfying (9) would lead to a contradiction:

$$
\begin{gathered}
\frac{\left\langle X_{2}, B_{i}\right\rangle}{\operatorname{Tr}\left(X_{2}\right)(1+\eta)} \leq \frac{1}{\alpha} \leq \frac{\left\langle X_{1}, B_{i}\right\rangle}{\operatorname{Tr}\left(X_{1}\right)(1-\eta)} \\
\Longrightarrow \quad 1+3 \eta=1+\zeta<\frac{\left\langle X_{2}, B_{i}\right\rangle / \operatorname{Tr} X_{2}}{\left\langle X_{1}, B_{i}\right\rangle / \operatorname{Tr} X_{1}} \leq \frac{1+\eta}{1-\eta}<1+3 \eta
\end{gathered}
$$

for sufficiently small $\eta$.

So the oracle must choose a matrix $B_{i, j}$ with $i=3$. In this case,

$$
\begin{gathered}
\frac{\operatorname{Tr} B_{i, j}}{\rho} \leq \frac{1}{\alpha} \leq \frac{\left\langle X_{1}, B_{i, j}\right\rangle}{\operatorname{Tr}\left(X_{1}\right)(1-\eta)} \\
\Longrightarrow \quad \frac{n}{9 \eta}=\frac{n}{3 \zeta} \leq \frac{\left(1+\zeta^{3}+\zeta\right) k}{\zeta}=\frac{\operatorname{Tr}\left(B_{i, j}\right) \operatorname{Tr}\left(X_{1}\right)}{\left\langle X_{1}, B_{i, j}\right\rangle} \leq \frac{\rho}{1-\eta} .
\end{gathered}
$$

This shows that $\rho=\Omega(n / \eta)$.

\section{The positive semidefiniteness assumption}

Proposition 31. For every positive integer $n$, there exist matrices $B_{1}, \ldots, B_{m} \in \mathbb{S}^{n}$ with $m=\Omega\left(n^{2}\right)$ such that $B:=\sum_{i} B_{i}$ is positive definite and with the following property: for every $\varepsilon \in(0,1)$ and $y \in \mathbb{R}^{m}$ such that $(1-\varepsilon) B \preceq \sum_{i} y_{i} B_{i}$, all entries of y are nonzero.

Proof. Let $\mathcal{P}:=\{(i, j): i, j \in[n], i<j\}$. For $(i, j) \in \mathcal{P}$, let $E_{i j}:=e_{i} e_{j}^{T}+e_{j} e_{i}^{T}$. Let $J$ denote the matrix of all ones. Then $2 I+\sum_{(i, j) \in \mathcal{P}} E_{i j}=I+J=: B \succ 0$. Let $\varepsilon \in(0,1)$ and suppose that $(1-\varepsilon) B \preceq 2 t I+\sum_{(i, j) \in \mathcal{P}} z_{i j} E_{i j}$ for some $t \in \mathbb{R}$ and $z \in \mathbb{R}^{\mathcal{P}}$. By taking the inner product with $E_{a b}$ on both sides, we see that $0<2(1-\varepsilon) \leq z_{a b}$ for every $(a, b) \in \mathcal{P}$. Similarly, we find that $0<2 n(1-\varepsilon) \leq 2 n t$. 